\title{
Wearable systems for shoulder kinematics assessment: a systematic review
}

\author{
Arianna Carnevale ${ }^{1}$, Umile Giuseppe Longo ${ }^{1 *}$ (D) Emiliano Schena ${ }^{2}$, Carlo Massaroni ${ }^{2}$, Daniela Lo Presti ${ }^{2}$, \\ Alessandra Berton ${ }^{1}$, Vincenzo Candela ${ }^{1}$ and Vincenzo Denaro ${ }^{1}$
}

\begin{abstract}
Background: Wearable sensors are acquiring more and more influence in diagnostic and rehabilitation field to assess motor abilities of people with neurological or musculoskeletal impairments. The aim of this systematic literature review is to analyze the wearable systems for monitoring shoulder kinematics and their applicability in clinical settings and rehabilitation.

Methods: A comprehensive search of PubMed, Medline, Google Scholar and IEEE Xplore was performed and results were included up to July 2019. All studies concerning wearable sensors to assess shoulder kinematics were retrieved.

Results: Seventy-three studies were included because they have fulfilled the inclusion criteria. The results showed that magneto and/or inertial sensors are the most used. Wearable sensors measuring upper limb and/or shoulder kinematics have been proposed to be applied in patients with different pathological conditions such as stroke, multiple sclerosis, osteoarthritis, rotator cuff tear. Sensors placement and method of attachment were broadly heterogeneous among the examined studies.

Conclusions: Wearable systems are a promising solution to provide quantitative and meaningful clinical information about progress in a rehabilitation pathway and to extrapolate meaningful parameters in the diagnosis of shoulder pathologies. There is a strong need for development of this novel technologies which undeniably serves in shoulder evaluation and therapy.
\end{abstract}

Keywords: Shoulder kinematics, Upper limb, Wearable system, Inertial sensors, Smart textile

\section{Background}

Shoulder kinematics analysis is a booming research field due to the emergent need to improve diagnosis and rehabilitation procedures [1]. The shoulder complex is the human joint characterized by the greatest range of motion (ROM) in the different planes of space.

Commonly, several scales and tests are used to evaluate shoulder function, e.g., the Constant-Murley score (CMS), the Simple Shoulder test (SST), the Visual Analogue Scale (VAS) and the Disability of the

\footnotetext{
*Correspondence: g.longo@unicampus.it

'Department of Orthopaedic and Trauma Surgery, Campus Bio-Medico

University, Via Álvaro del Portillo, 200, 00128 Rome, Italy

Full list of author information is available at the end of the article
}

Arm, Shoulder, and Hand (DASH) score [2-4]. However, despite their easy-to-use and wide application in clinical settings, these scores conceal an intrinsic subjectivity [2-4], inaccuracy in approaching diagnosis, follow-up and treatment of the pathologies. Quantitative and objective analyses are rapidly developing as a valid alternative to evaluate shoulder activity level, to gauge its functioning and to provide information about movement quality, e.g., velocity, amplitude and frequency $[5,6]$. This interest in the use of measuring systems is growing in many medical fields to record information of clinical relevance. For example, electromyography (EMG), force sensors, inertial measurement units (IMU), accelerometers, fiber optic sensors and strain sensors are employed for human motion analysis, posture and physiological parameters monitoring [7-10]. From a technological viewpoint, the

(c) The Author(s). 2019 Open Access This article is distributed under the terms of the Creative Commons Attribution 4.0 International License (http://creativecommons.org/licenses/by/4.0/), which permits unrestricted use, distribution, and reproduction in any medium, provided you give appropriate credit to the original author(s) and the source, provide a link to the Creative Commons license, and indicate if changes were made. The Creative Commons Public Domain Dedication waiver (http://creativecommons.org/publicdomain/zero/1.0/) applies to the data made available in this article, unless otherwise stated. 
monitoring of shoulder motion is challenging due to the complexity of joint kinematic which require the development of protocols exploiting sensing technology as much as possible reliable and unobtrusive. In the last years, a great number of human motion analysis systems have been largely employed for objective monitoring. These systems can be classified into two main categories: wearable and non-wearable [11]. The last one includes electromagnetic tracking systems (e.g., Fastrak) [12], ultrasound-based motion analysis systems (e.g., Zebris) [13], stereo-photogrammetric and optoelectronic systems (e.g., VICON, Optotrak, BTS SMART-D) often used as gold standard [14-17]. These systems based on magnetic field, ultrasound and cameras are effectively suitable for 3D motion tracking and analysis due to their accuracy, precision and reliability [18]. On the other hand, such systems require expensive equipment, frequent calibration and, overall, they restrict measurements in structured environment [19]. Wearable systems overcome these shortcomings and they are a promising solution for continuous and long-term monitoring of human motion in daily living activities. Gathering data in unstructured environment continuously (e.g., home environment) provide additional information compared to those obtainable inside a laboratory [20].

Wearable sensor-based systems, intended for kinematics data extraction and analyses, are acquiring more and more influence in diagnostic applications, rehabilitation follow-up, and treatments of neurological and musculoskeletal disorders [21, 22]. Such systems comprise accelerometers, gyroscopes, IMU, among others [23]. Patients' acceptance of monitoring systems that should be worn for long-time relies on sensors' features whose must be lightweight, unobtrusive and user-friendly [24]. The increasing trend to adopt such wearable systems has been promoted by the innovative technology of micro-electro-mechanical systems (MEMS). MEMS technology has fostered sensors' miniaturization, paving the way for a revolutionary technology suited to a wide range of applications, including extraction of clinical-relevant kinematics parameters. In recent years, there has been growth in the use of smart textile-based systems which integrate sensing units directly into garments $[11,25,26]$. Moreover, in the era of big data, machine learning technical analysis can improve home rehabilitation thanks to the recognition of the quality level of performed physical exercises and the possibility to prevent disorders in patients' movement [27].

The aim of this systematic literature review is to describe the wearable systems for monitoring shoulder kinematics. The authors want to summarize the main features of the current wearable systems and their applicability in clinical settings and rehabilitation for shoulder kinematics assessment.

\section{Methods \\ Literature search strategy and study selection process}

A systematic review was executed applying the PRISMA (Preferred Reporting Items for Systematic Reviews and Meta-Analyses) guidelines [28]. Full-text articles and conference proceedings were selected from a comprehensive search of PubMed, Medline, Google Scholar and IEEE Xplore databases. The search strategy included free text terms and Mesh (Medical Subject Headings) terms, where suited. These terms were combined using logical Boolean operators. Keywords and their synonyms were combined in each database as follows: "shoulder biomechanics" OR "upper extremit" " OR "shoulder joint" OR "scapular-humeral" OR "shoulder kinematics" OR "upper limb") AND ("wearable system" OR "wearable device" OR "wearable technolog*" OR "wearable electronic device" " OR "wireless sensor" OR "sensor system" OR "textile" OR "electronic skin" OR "inertial sensor"). No filter was applied on the publication date of the articles, and all results of each database were included up to July 2019. After removal of duplicates, all articles were evaluated through a screening of title and abstract by three independent reviewers. The same three reviewers performed an accurate reading of all full-text articles assessed for eligibility to this study and they performed a collection of data to minimize the risk of bias. In case of disagreement among investigators regarding the inclusion and exclusion criteria, the senior investigator made the final decision.

Inclusion criteria were:

i) The studies concern wearable systems as a tool to assess upper limb kinematics;

ii) The studies used sensors directly stuck on the human skin by means of adhesive, embedded within pockets, straps or integrated into fabrics;

iii) Systems intended for motion recognition and rehabilitation;

iv) Articles are written in English language;

v) Papers are published in a peer-reviewed journal or presented in a conference;

Exclusion criteria were:

i) Use of prosthetics, exoskeleton or robotic systems;

ii) Wearable system not directly worn or tested on human;

iii) The study concerns wearable systems for full-body motion tracking; 
iv) Shoulder joint is not included;

v) Reviews, books.

\section{Data extraction process}

Data extraction was executed on 73 articles. Data was extracted on the base of the following checklist: authors, year and type of publication (i.e., conference or full-text); typology, number, brand and placement of the sensors used to measure or track the kinematic of the interested joint, wearability of the system, target parameters with regard to the shoulder; system used as gold standard to assess the wearable systems' performance; tasks executed in the assessment protocol; characteristics of the participants involved in the study and aim of the study.

\section{Results}

The literature search returned 1811 results and additional 14 studies were identified through other sources. A total of 73 studies fulfilled the inclusion criteria (Fig. 1), of which $27 \%$ were published on conference proceedings and the remaining $73 \%$ on peer-reviewed journal.

Three levels of analysis have been emphasized in this survey: A. application field and main aspects covered, B. the typology of sensors exploited to measure kinematic parameters, $\mathrm{C}$. the placement of the single measurement units on the body segment of interest and how sensing modules are integrated into the wearable system from a wearability viewpoint.

\section{Application field}

Fifteen out of the 73 studies focused on evaluating upper limbs motion in case of musculoskeletal diseases (e.g., osteoarthritis, rotator cuff tear, frozen shoulder), 26 on neurological diseases and ap-plication in neurorehabilitation (e.g., stroke, multiple sclerosis), 15 on general rehabilitation aspects (e.g., home rehabilitation, physiotherapy monitoring) and 17 focusing on validation and development of systems and algorithm for monitoring shoulder kinematics. Tables 1, 2, 3 and 4 include, for each of the identified application fields, data listed in the previous data extraction process section.

\section{Sensing technology}

Some studies combined different sensors in their measurements system. The most used sensors are accelerometers, gyroscopes and magnetometers, a combination

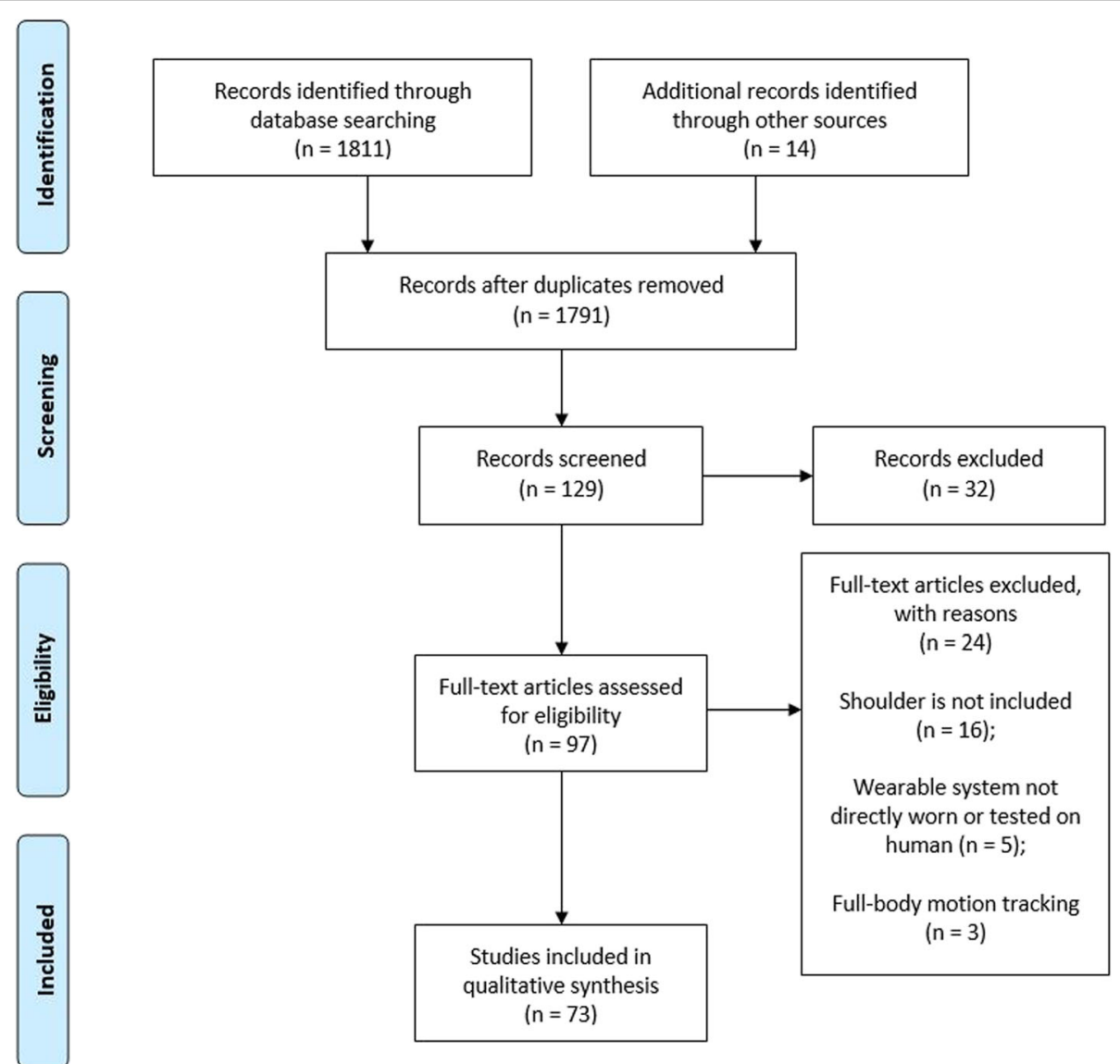

Fig. 1 PRISMA 2009 flow diagram 
Carnevale et al. BMC Musculoskeletal Disorders

(2019) 20:546

Page 4 of 24

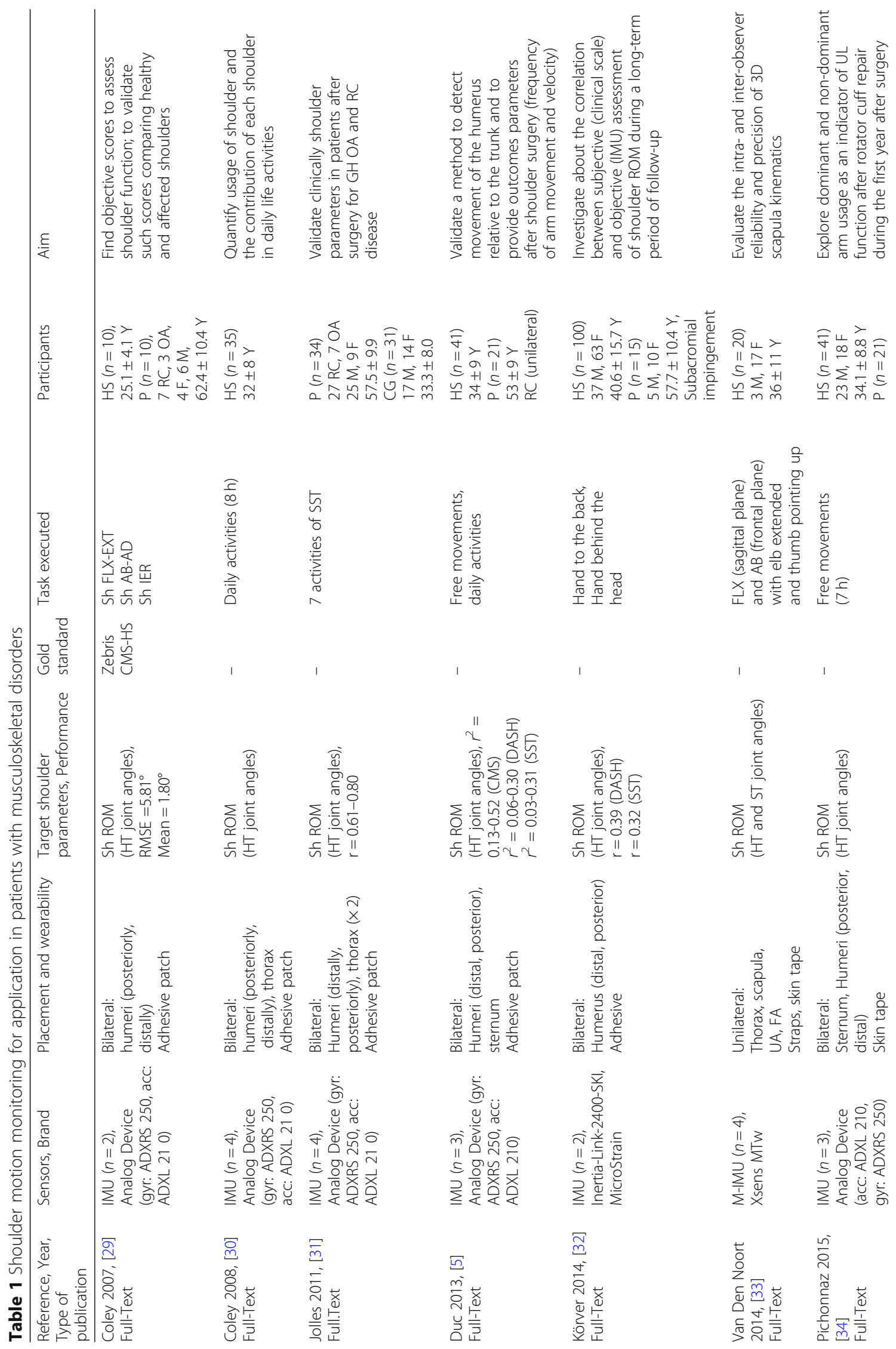




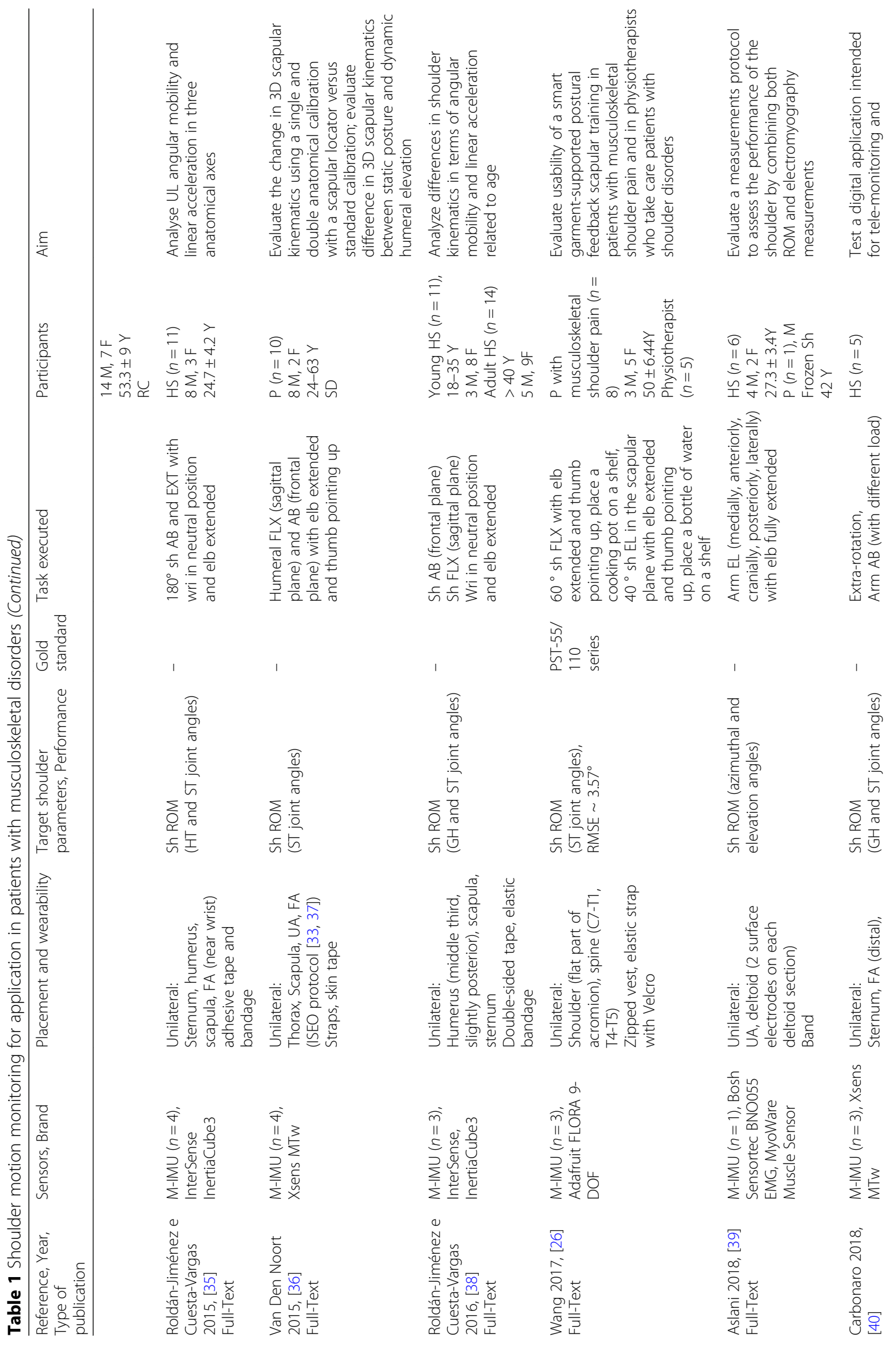




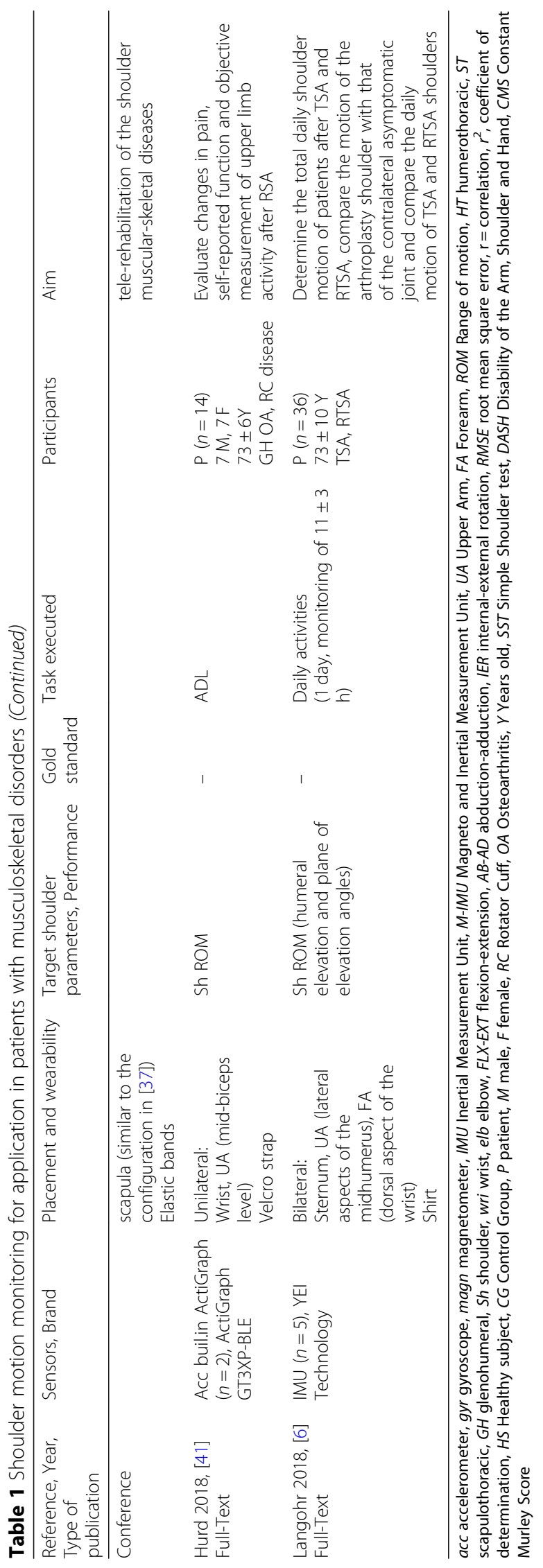




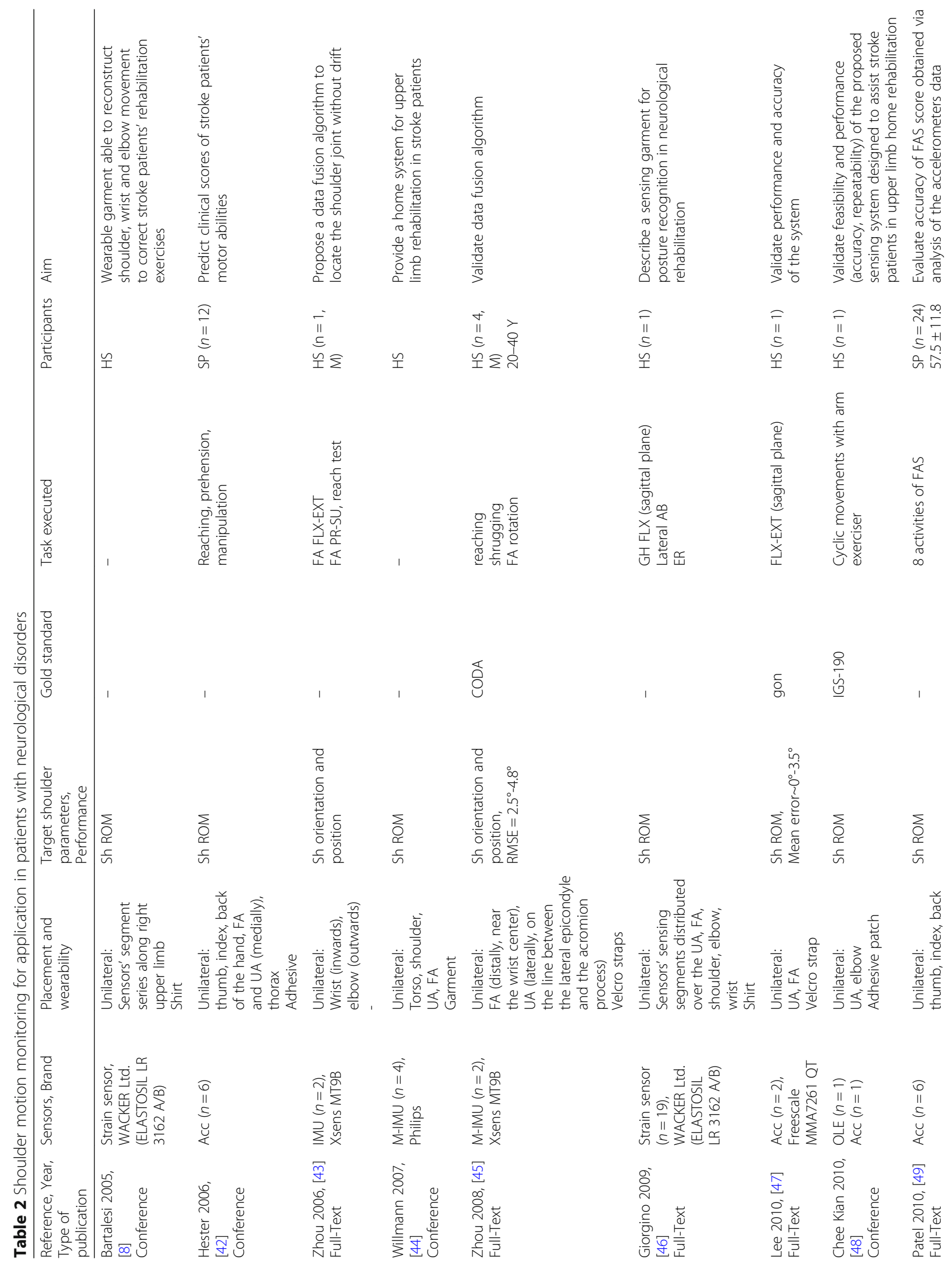




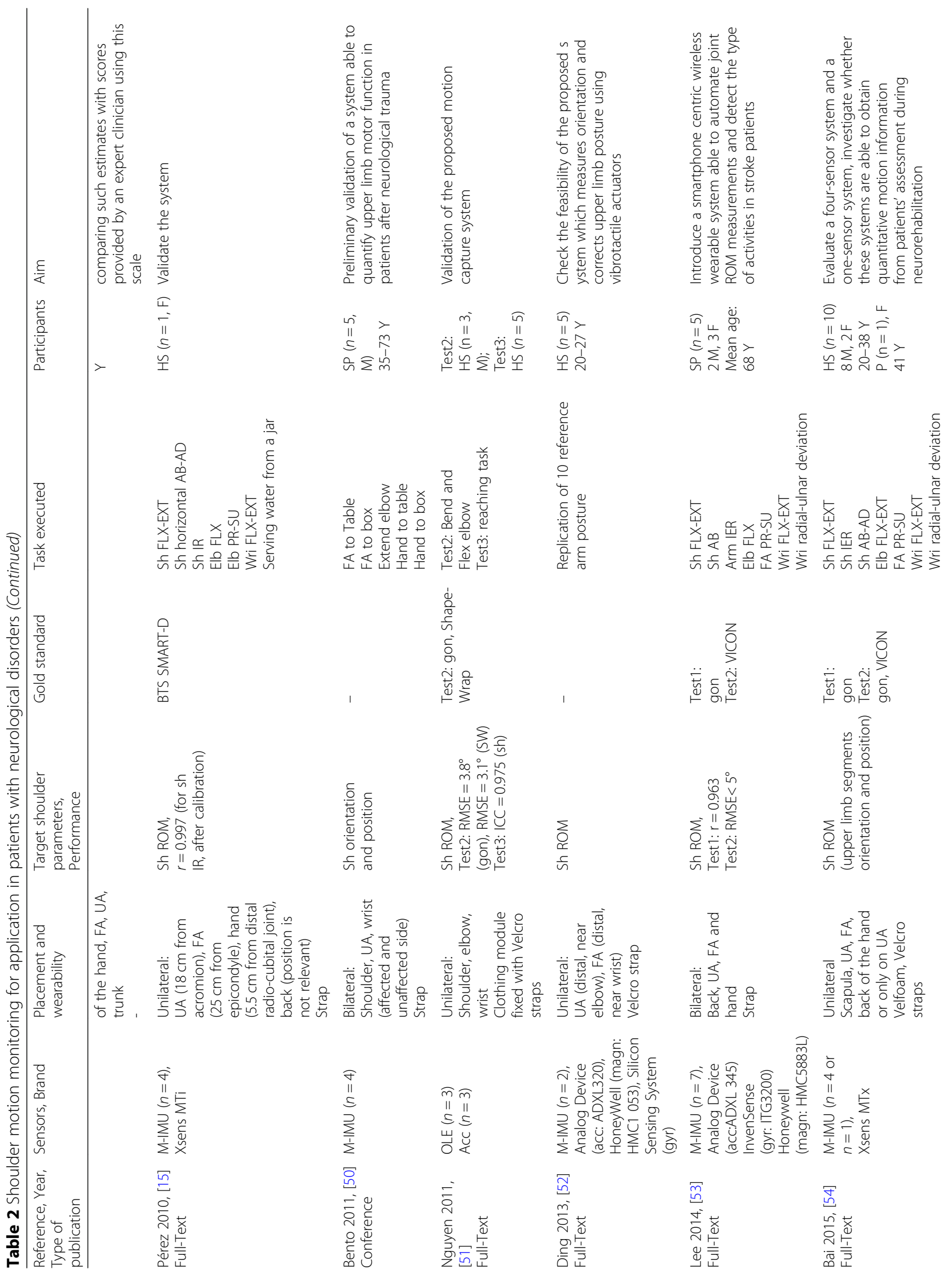




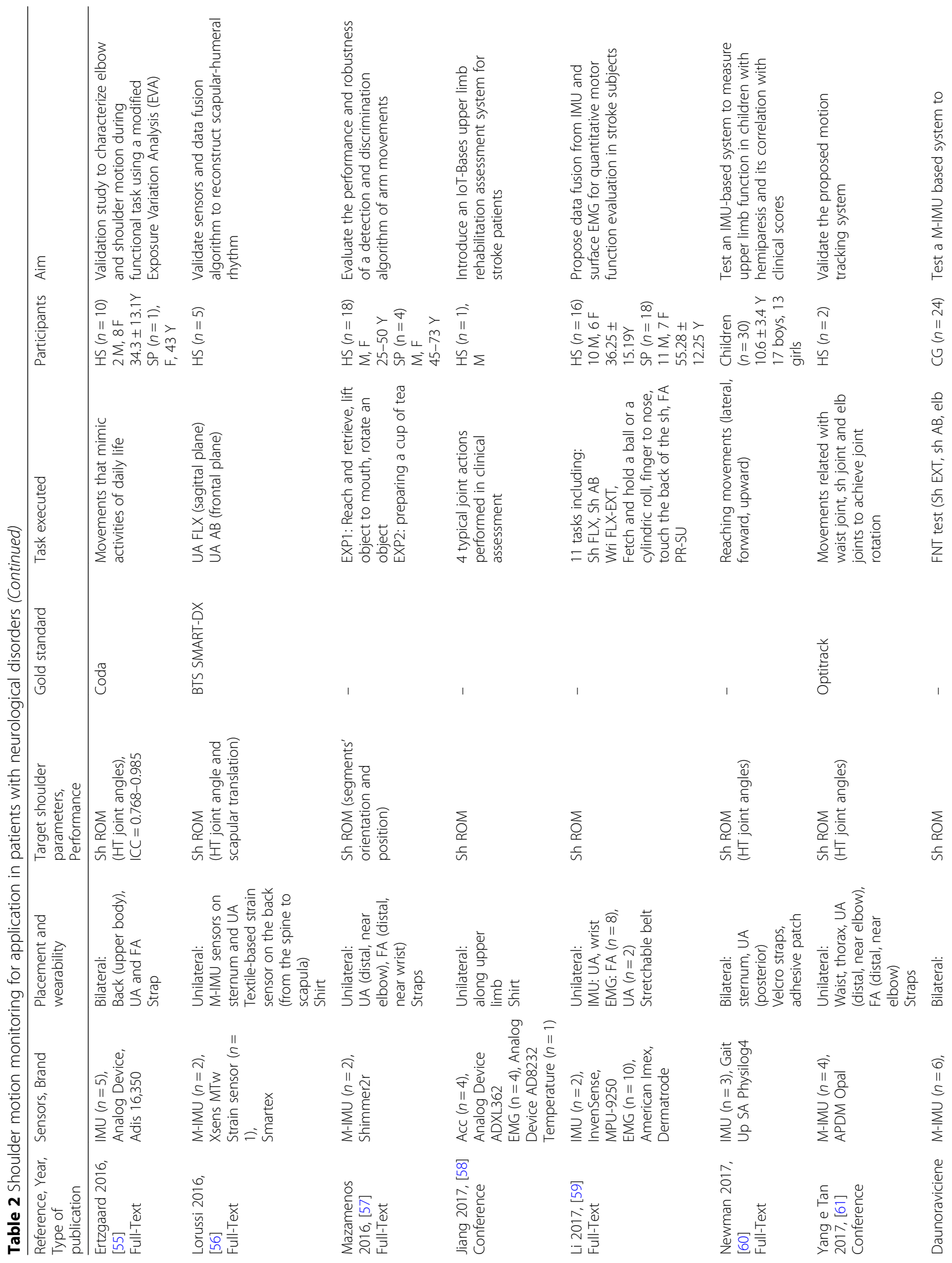




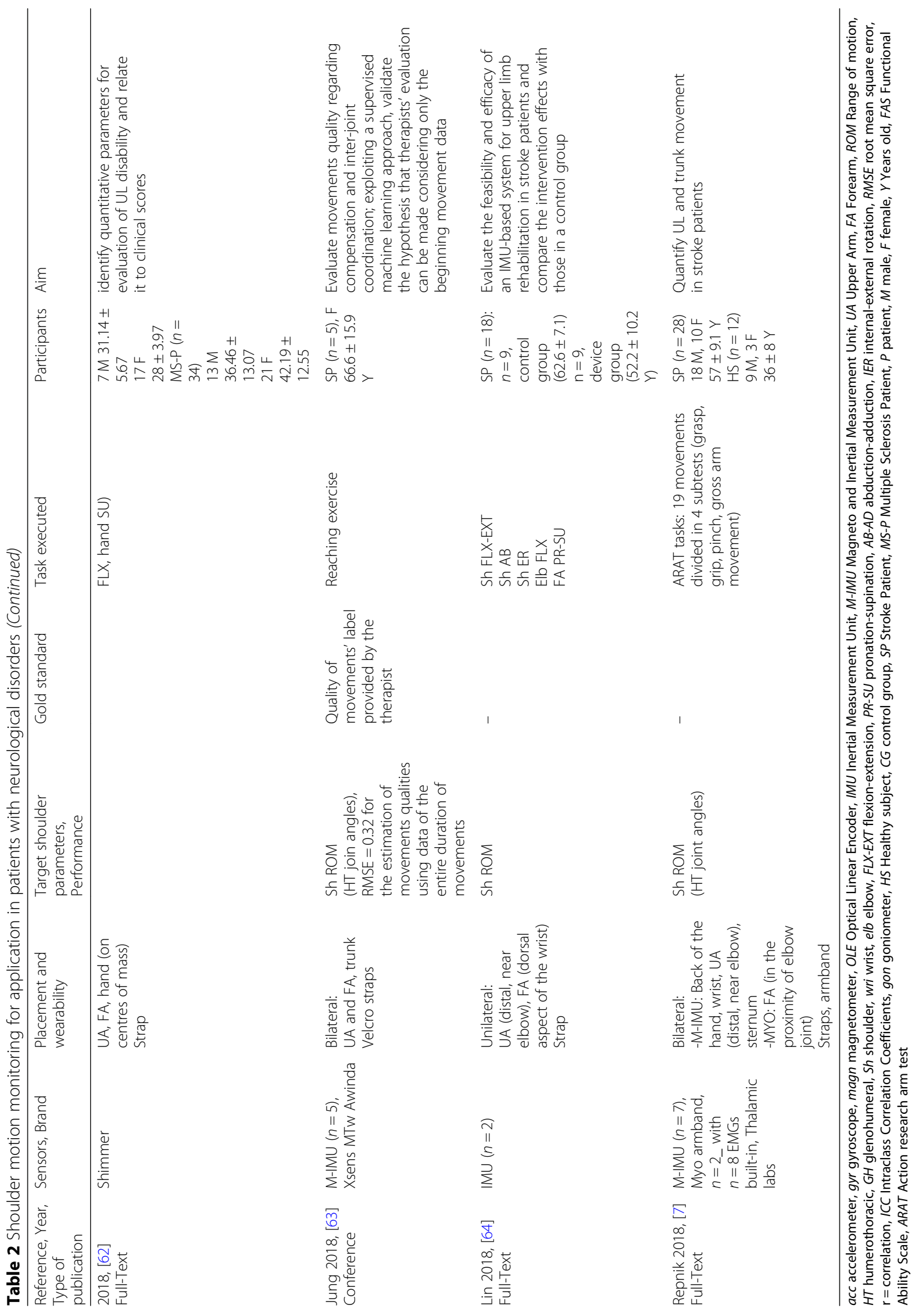




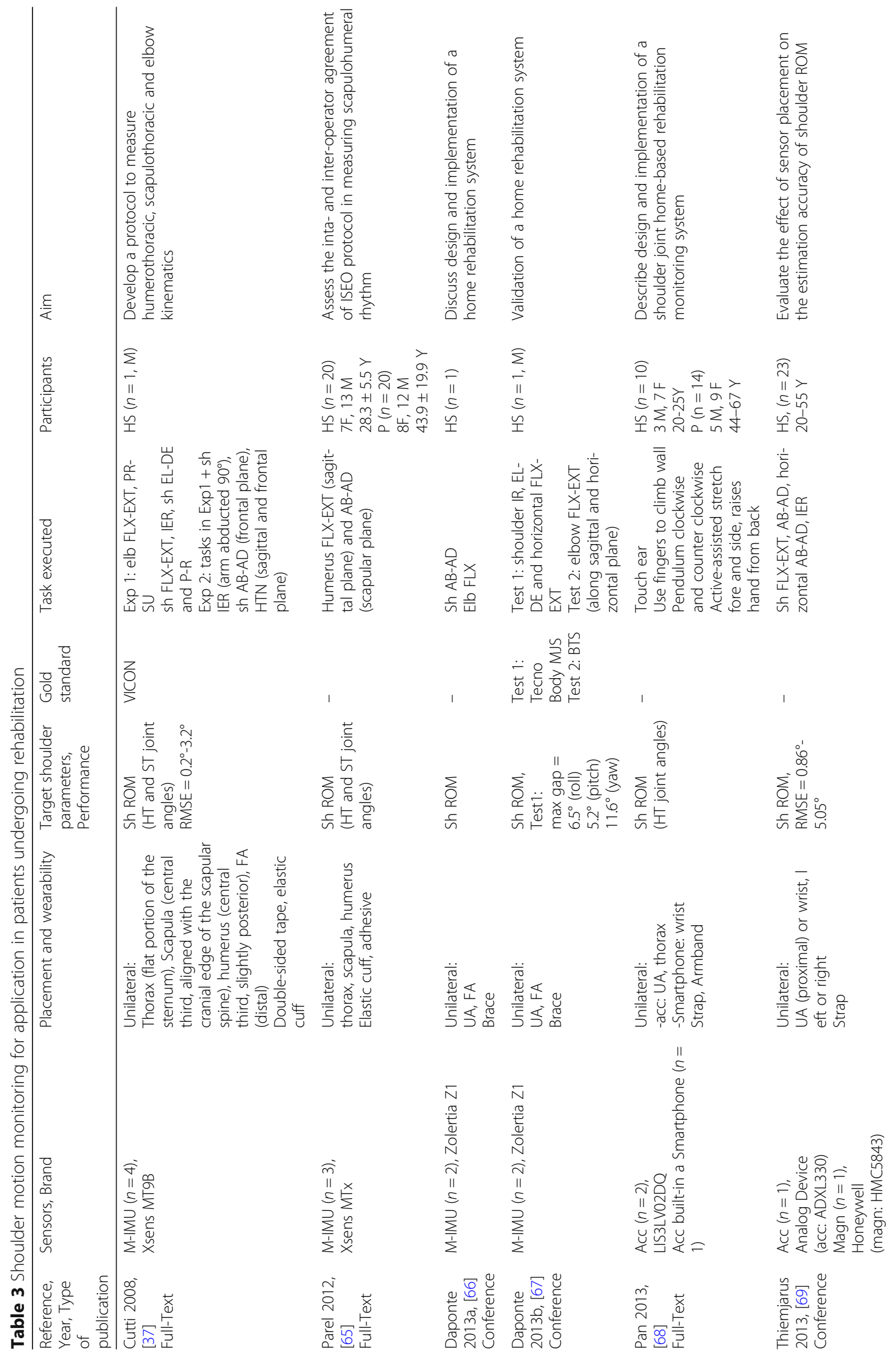


Carnevale et al. BMC Musculoskeletal Disorders (2019) 20:546

Page 12 of 24

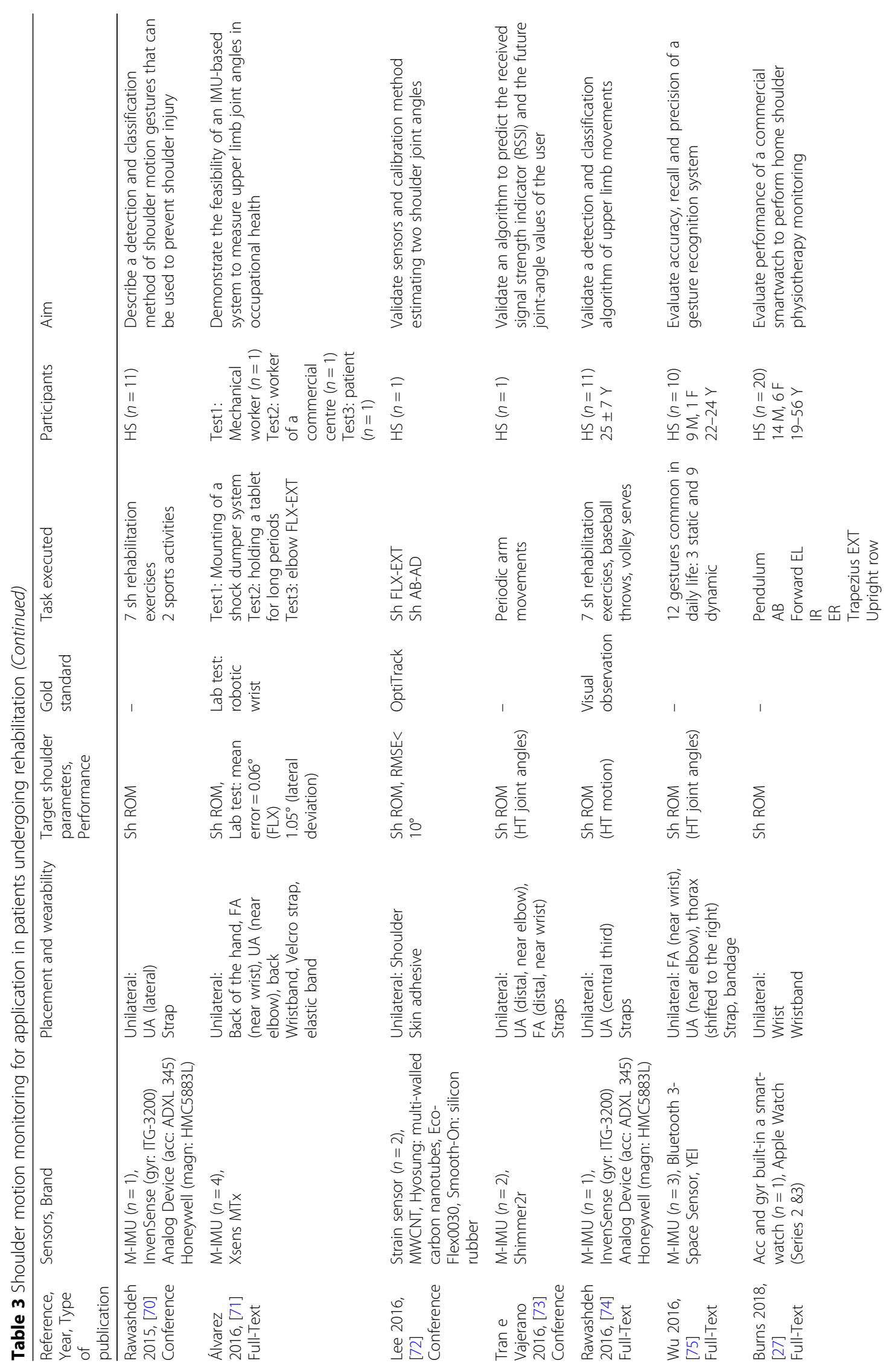


Carnevale et al. BMC Musculoskeletal Disorders (2019) 20:546

Page 13 of 24

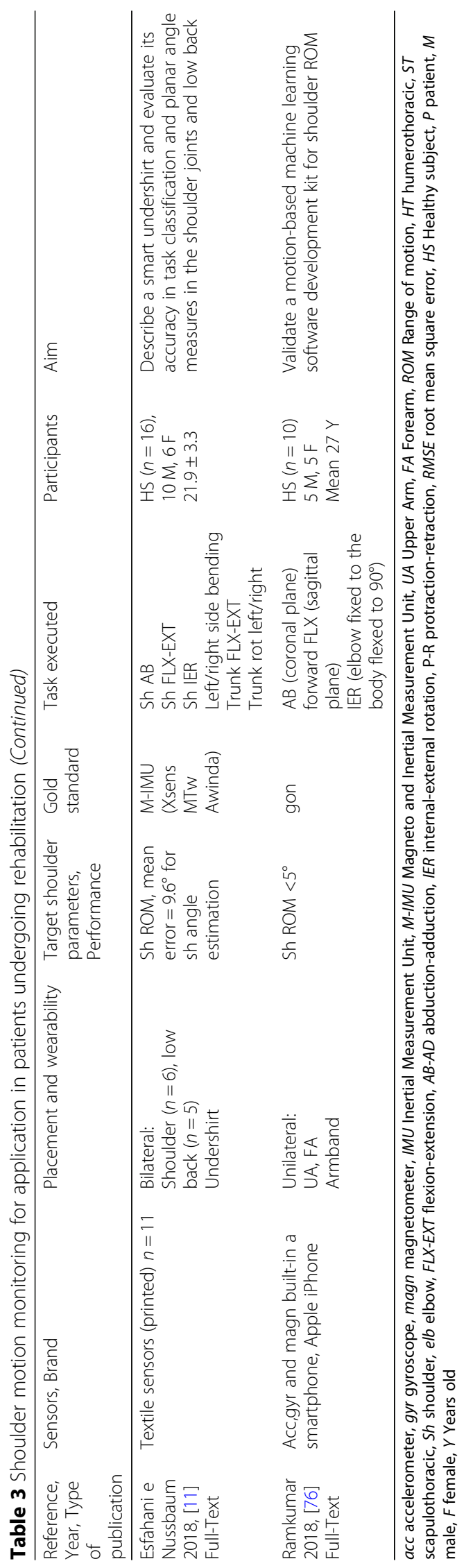




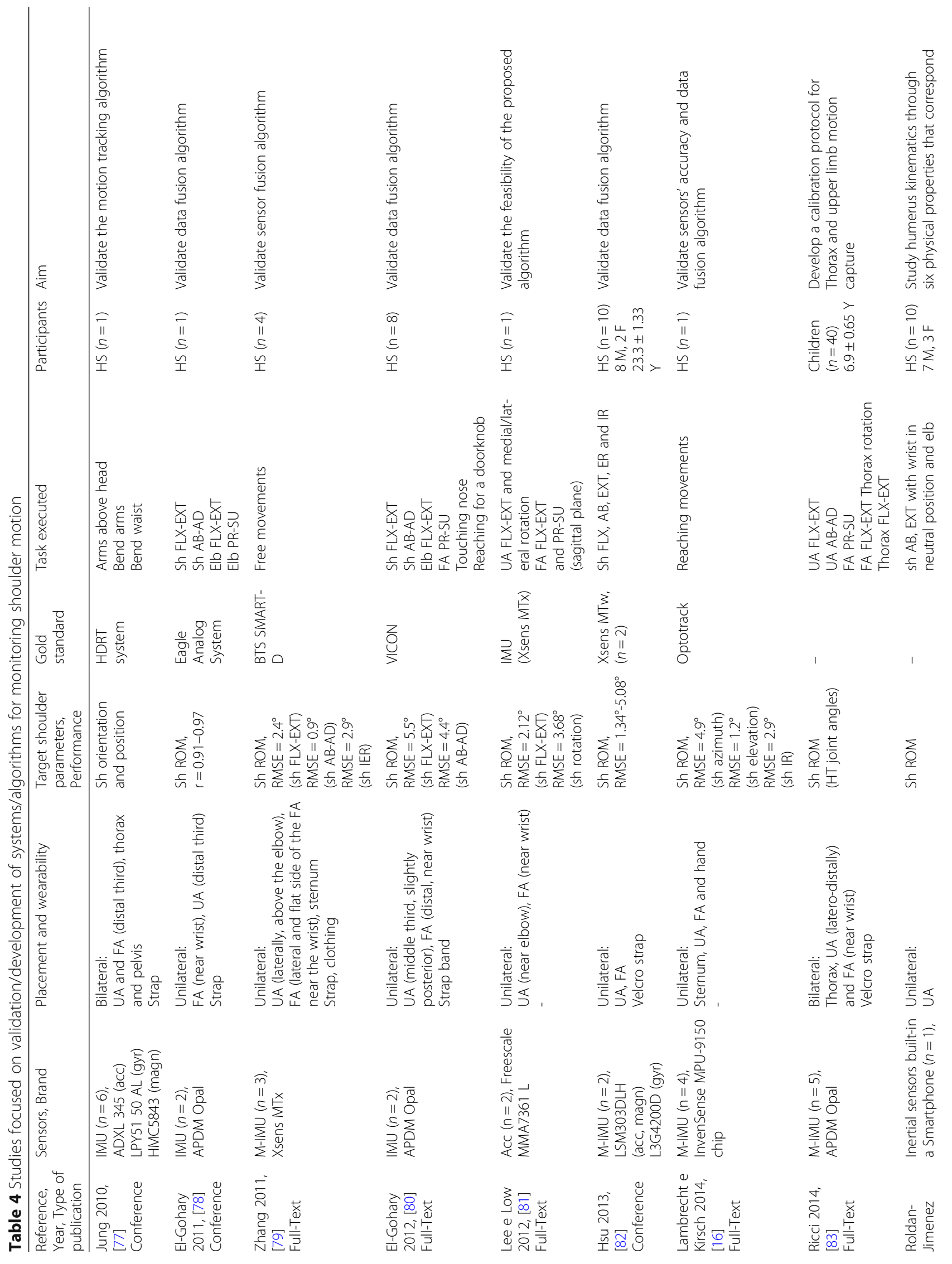




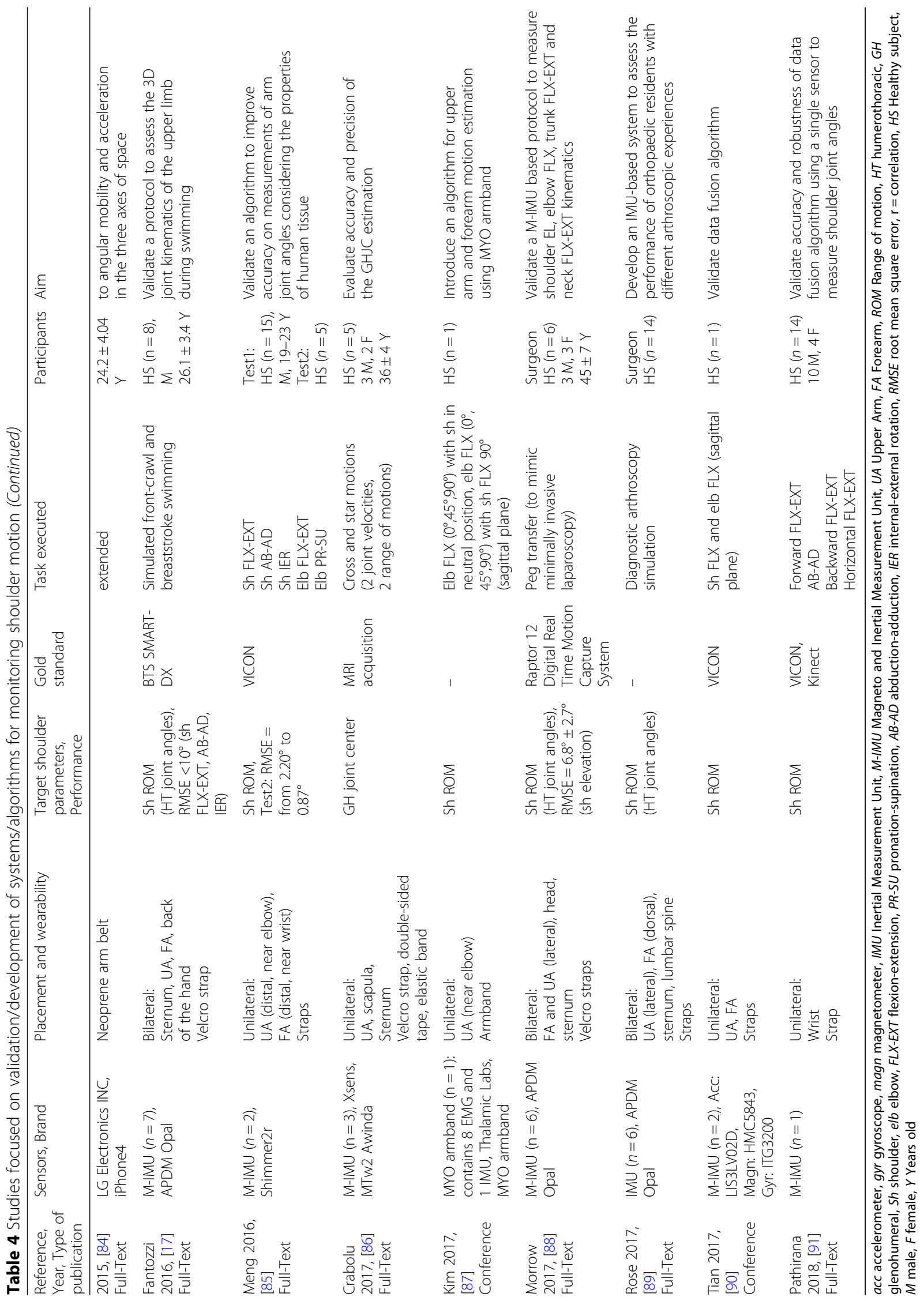


of them $(n=55)$ or with other sensors $(n=8)$, or built-in into other devices (e.g., smartphones, smartwatch) $(n=$ $6)$; additional studies $(n=4)$ utilized strain sensors for motion analysis.

\section{B.1 wearable systems based on inertial sensors and magnetometers}

An IMU allows estimating both translational and rotational movements. Such sensors comprise gyroscopes that measure angular velocity and accelerometers that measure proper acceleration, i.e. gravitational force (static) and force due to movements (dynamic) [92]. The main limitation of the gyroscopes is the issue bias due to drift. Gyroscopes do not have an external reference, as opposed to accelerometers that use gravity vector as reference; in the orientation estimation, gyroscopes suffer of drift during the integration procedures. To compensate such issue, these sensors are combined with magnetometers that measure magnetic field and use the Earth's magnetic field as reference. The main limitation of magnetometers is the interference due to the presence of ferromagnetic materials in the surrounding environment [92]. We refer to these hybrid sensors as M-IMU (magnetic and inertial measurement unit). By integrating the information derived from each sensor (i.e., acceleration, angular velocity and magnetic field) through sensorfusion algorithms, M-IMUs provide an accurate estimation of the 3D-position and 3D-orientation of a rigid body. The upper limb can be modelled as a kinematic chain constituted by a series of rigid segments, i.e., thorax, upper arm, forearm and hand, linked to each other by joints that allow relative motion among consecutive links [17]. In the kinematic chain, the shoulder joint consists of three degrees of freedom (DOFs) correspondent to abduction-adduction (AB-AD), internal-external rotation (IER), and flexion-extension (FLX-EXT) [15, 54, 57, 71, 79]. Shoulder rotations can be described using Euler angles that identify the anatomical DOFs with the roll-pitch-yaw angles [17, 33, 37, 88]. Sensor-fusion algorithms can exploit two main approaches, deterministic or stochastic. The deterministic approach includes the complementary filter that merges a high pass filter for gyroscope data (to avoid drift) and a low pass filter for accelerometer and magnetometer data $[64,82,90,92]$. The stochastic approach includes the Kalman Filter and its more sophisticated versions [7, $55,66,67,78-80,91,92]$. The Kalman filter (KF) is the most used algorithm to process M-IMU and IMU data due to its accuracy and reliability $[15,38,54,75,83,93]$.

Wearable systems based on IMU or M-IMU include a variable number of sensor nodes that, properly distributed on each body segment of interest, provide kinematic parameters such as joint ROM, position, orientation, and velocity. Fifty-one out of the included studies used exclusively IMUs $(n=15)$ or M-IMUs $(n=$ 36). Systems performances were analyzed in terms of the agreement between results obtained from the M-IMU or IMU-based systems and those collected by a gold standard system. Several types of systems were used as gold standard, such as ultrasound-based system (e.g., Zebris CMS-HS [29]), diagnostic imaging (e.g., Magnetic Resonance [86]), optical-based systems (e.g., VICON [37, 53, 54, 80, 85, 90, 91], BTS Bioengineering [15, 17, 56, 67, 79], Eagle Analogue System [78], Optotrack [16], Optitrack [61], CODA [45, 55]), goniometer [53, 54]. Results from an inertial system were benchmarked against an ultrasound-based reference system, showing a root mean square error (RMSE) of $5.81^{\circ}$ and a mean error of $1.80^{\circ}$ in the estimation of shoulder angles of FLX-EXT, $A B-A D$ and IER evaluated in the sagittal, frontal and transversal planes, respectively [29]. Accuracy of a protocol based on commercial inertial sensors (MT9B, Xsens) was tested and compared to a VICON system to measure humerothoracic, scapulothoracic joint angles and elbow kinematics [37]. Results demonstrated high accuracy in the estimation of upper limb kinematics with an RMSE lower than $3.2^{\circ}$ for $97 \%$ of data pairs. A BTS reference system was used to validate accuracy of a wearable system comprised of commercial sensors (Xsens) and results showed a mean error difference of $13.82^{\circ}$ for FLX-EXT, $7.44^{\circ}$ for $\mathrm{AB}-\mathrm{AD}, 28.88^{\circ}$ for IR [15]. In a protocol-validation study, commercial Opal sensors were compared to a BTS system to assess upper limb joint kinematics during simulated swimming movements. Data showed a median RMSE always better than $10^{\circ}$ considering movements of AB-AD, IER and FLX-EXT in front-crawl and breaststroke [17]. Opal wearable sensors were compared to optical motion capture systems to estimate shoulder and elbow angles [78, 80]. Planar shoulder FLX-EXT and AB-AD were performed showing an RMSE of $5.5^{\circ}$ and $4.4^{\circ}$, respectively [80]; a good correlation between the measurements performed on shoulder motion with the two systems was also found in [78] (no data regarding measurements error were proposed).

Some studies $(n=11)$ compared data obtained from wearable sensors, custom or commercial, with a gold standard to validate their own sensors data fusion algorithm (for more details see Table 4). Two different algorithms were compared to a customized KF [79]. Comparing the results derived from the BTS system and the inertial-based system (Xsens), the proposed algorithm showed a smaller error than the other two methods for computing shoulder FLX-EXT $($ RMSE = $\left.2.4^{\circ}\right), \mathrm{AB}-\mathrm{AD}\left(\mathrm{RMSE}=0.9^{\circ}\right)$, IER $\left(\mathrm{RMSE}=2.9^{\circ}\right)$ [79]. The addition of the magnetometer-based heading correction in the sensor data fusion algorithm was investigated to test the accuracy of an inertial-based motion tracking system using the Optotrak Certus (Northern Digital Inc., 
Waterloo, ON, Canada) as reference. Results showed a RMSE of $4.9^{\circ}, 1.2^{\circ}$ and $2.9^{\circ}$ for shoulder azimuth, elevation and internal rotation, respectively [16].

Four studies used only accelerometers [42, 47, 49, 81]. Systems performance analysis in measurement of arm motion, showed a RMSE lower than $3.5^{\circ}$ and $3.68^{\circ}$ for shoulder ROM when results from the accelerometersbased systems were benchmarked against a goniometer and commercial M-IMUs, respectively [47, 81]. Evaluation of upper limbs' physical activity was performed recording data of accelerometers built-in wearable device as ActiGraph (Pensacola, Florida, Model GT3XP-BTLE) to obtain objective outcomes in patients after reverse shoulder arthroplasty [41].

Shoulder ROM has been also estimated by means of a single sensor node which integrated an accelerometer and a magnetometer [69]. Sensor fusion algorithms of accelerometers and magnetometers data provide accurate orientation estimation in static or semi static condition, e.g., in a rehabilitation session in which patients perform slow movements [81]. M-IMUs comprised of a $3 \mathrm{D}$ accelerometer, 3D gyroscope and 3D magnetometer are the most appropriate choice for motion tracking either in static that in dynamic condition.

Two accelerometer-based sensors were combined with those built-in a smartphone to realize a smart rehabilitation platform for shoulder home-rehabilitation [68]. Mobile phone or a smartwatch, with their built-in inertial sensor units, were used as mobile monitoring devices [27, $76,84]$. These results give proof of the growing trend in the application of commercial devices in clinical setting for rehabilitation purposes. Data has been processed using machine learning algorithms to extract salient features and for gesture recognition related to shoulder motion. In these techniques, the main steps are the data collection, followed by segmentation process, feature extraction and classification [27, 49]. For instance, the identification of different types of RC physiotherapy exercises has been performed processing data from inertial sensors built-in a wrist-worn smartwatch [27]. Data from inertial sensors built-in a smartphone were benchmarked against a manual goniometer. Angular differences between a machine learning-based application and goniometer measurements resulted less than $5^{\circ}$ for all shoulder ROM (i.e., $\mathrm{AD}$, forward FLX, IR, ER) [76].

Two studies combined accelerometer(s) with Optical Linear Encoder (OLE) [68, 84]. An OLE-based system acts as a goniometer providing measures of joint angles. Despite of the simplicity and low cost of the proposed systems, differences in shoulder ROM estimation resulted not negligible when data collected by the wearable systems were compared against an inertial-based motion capture (i.e., IGS-190 [54]) and a fiber optics-based system (i.e., ShapeWrap [71]).
Three studies included EMG sensors in their assessment tool in combination with accelerometers [58], IMUs [59] and M-IMU [39]. EMG sensors placed on the biceps, triceps [59] and deltoid muscles [39] provide additional information about upper limb motor function and shoulder assessment, evaluating muscles activity. Quantification of upper limb motion was executed through a wearable device, MYO armband by Thalamic labs, that combines EMG sensors to record electrical impulses of the muscles [7, 87].

\section{B.2 wearable systems based on strain sensors}

Four studies used smart-textiles instrumented by strain sensors with piezoresistive properties to estimate kinematic parameters and to perform motion analysis $[8,11$, $46,56]$. Such sensing elements are stretched or compressed during movements of the examined body segments, with consequent variation of their electrical resistance [94, 95]. Using a M-IMU system as reference, accuracy evaluation of a smart-textile with printed strain sensors showed a mean error of $9.6^{\circ}$ in planar motions measurements of shoulder joint [11]. Shoulder kinematics was assessed combining a strain sensor for scapular sliding detection with two M-IMUs for HT orientation measure [56].

Piezoresistive strain sensors directly adhered to the skin were used to estimate shoulder ROM; the comparison between reference data from an optical-based system (i.e., Optitrack) and strain sensors showed a RMSE less than $10^{\circ}$ in shoulder FLX-EXT and AB-AD estimation [72].

\section{Sensors placement and wearability}

Placement of the sensing technology on the body landmarks has shown a heterogeneous distribution linked to the different nature of the employed technology and to the purpose for which monitoring system was designed. With respect to the monitored upper limb, 53 out of the 73 studies included in this review showed a unilateral distribution of the sensing elements while the remaining studies utilized a bilateral placement. Several configurations using different number of sensors and placements have been investigated as reported in detail in each table and Fig. 2.

Regarding the wearability, we classified the systems in terms of how the sensors were fixed to the human body: i) by adhesive patch, ii) by means of straps or embedded within pocket, iii) the sensing element is physically integrated into the fabric. Four studies did not specify the method of attachment, 12 studies have stuck sensors directly on human skin by means of adhesive patch, 52 studies have attached sensors through straps or embedding them in modular clothing, and 5 studies have integrated sensors directly into garments. For more details refers to Tables $1,2,3$ and 4 . 


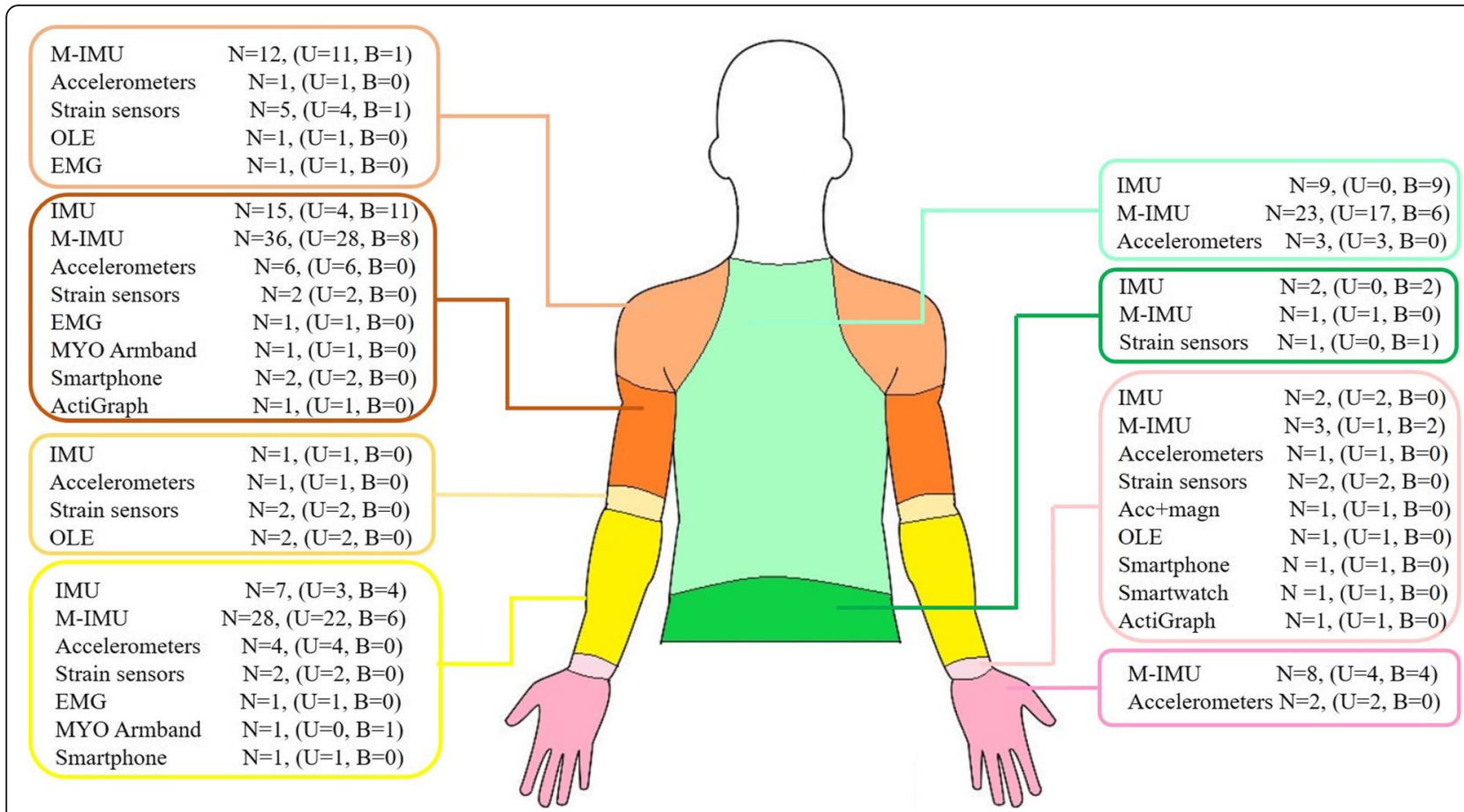

Fig. 2 Placement of sensing units (NOTE One study [90] is not included because the specific position of each sensor nodes is not so clear. Legend: $\mathrm{N}$ = number of studies, $\mathrm{U}=$ Unilateral, $\mathrm{B}=$ Bilateral)

\section{Discussion}

This paper summarizes the main features of wearable systems that have been employed in clinical setting and research field to evaluate upper limb functional performance and particularly for shoulder ROM assessment. Shoulder complex is characterized by the greatest mobility among all human joints and, due to its complexity, reviewed articles evidenced heterogeneity on the more suitable protocol for capturing joint ROM [96].

\section{Wearable technology}

Although $73 \%$ of the reviewed papers use commercial products for tracking joint angles, many of these personalize the positioning of the sensors, the calibration methodology and the algorithms used to process the recorded data. This customization makes strenuous a direct comparison among protocols, especially if sensing units of different nature (e.g., M-IMU vs. strain sensors) are used to measure the same kinematic parameters, leaving still open the issue of the protocols' definition with general validity.

About studies using inertial-based motion tracking systems, most in this summary (88\%), calibration procedures before data acquisition and data processing represent a relevant issue about accuracy and reliability of the system. Typically, the M-IMUs are attached on the segment of interest to estimate its orientation, so the calibration is necessary to relate sensors' measurements to movements of the tracked body segment. Sometimes the manufacturer suggests how to perform calibration, e.g., positioning sensors on a flat surface $[15,35]$ to align coordinate system or assuming static anatomical position [65], as N-pose [79], to compute orientation differences between segments and sensors coordinates in order to obtain sensor-to-segment alignment [56]. Dynamic or functional anatomical calibration has also been performed in some studies, but the sequence of movements executed varied among these $[17,33,55,83]$. One interesting improvement that may be done to have a positive impact on the accuracy of inertial-based motion tracking systems, is to define a standard set of movements for the initial calibration and a standard method of data processing by which extrapolate kinematic parameters of high clinical relevance.

Some works have reported remarkable results in human motion tracking using e-textile sensors $[8,46]$. Technological improvements in the development of conductive elastomers allowed to integrate such strain sensors directly into garments making them comfortable and unobtrusive $[11,56]$. Although conductive elastomers ensure flexibility and performances comparable with those of the M-IMU sensors, the main limitations are the hysteresis, uniaxial measurements and nonnegligible transient time [56]. Wearable systems based on strain sensors are a promising technology for kinematics analysis that may overcome the main M-IMUs 
drawbacks, as interferences due to surrounding ferromagnetic materials, gyroscopes' error drift and longterm use. On the other hand, errors may occur with strain sensors-based systems in the estimation of shoulder kinematics for their inherent hysteresis behaviour.

Among wearable systems reviewed in this summary, differences resulted in terms of sensors typology, number and size, placement, and wearability features. Sensors placement and method of attachment must be carefully investigated as they could influence the outcomes reliability (e.g., effects of soft tissues' artefacts). Human skeleton is covered by skin tissue and muscles. The combination of skin's elasticity and muscle activity may cause negative effects in the measurement of the bones' movement. In studies where M-IMU sensors were used to track shoulder kinematics, soft tissue properties were opportunely included in mathematical models to reduce soft tissue artifacts $[79,85]$. The body fat percentage was found the main influencing factor that negatively affects the inertial sensors' orientation [85]. To reduce such source of error, either when sensors are directly adherent to the skin that embedded in a textile, sensing units should be placed as near as possible to the bone segment to reduce soft tissue artifacts [97, 98]. Wearability is a key factor to consider because it can influence the level of patients' acceptance [26].There are several relevant requirements that wearable systems must meet to encourage their applications in continuous monitoring of patient status. Indeed, execution of movements, either in home environments or in clinical settings, should not be hindered by measurements systems so they must be non-invasive, modular, lightweight, unobtrusive and include a minimal number of sensors [33, $40,51,56,66,67,91]$. Most studies have employed magneto and inertial-based tracking systems in which sensors were attached to the upper limb through Velcro straps or including them in modular brace and garments $[26,45,67,82,88]$.

Upper limb includes the shoulder, elbow and wrist joints (Fig. 3a). Humerus, scapula, clavicle, and thorax constitute the shoulder complex: humeral head articulates in the glenoid fossa of the scapula to form $\mathrm{GH}$ joint, the AC joint is the articulation between the lateral end of the clavicle and the acromion process, the SC joint articulates the medial end of the clavicle and the sternum and the functional ST joint allows rotational and translational movements of the scapula with respect to the thorax [96] (Fig. 3b). The ST joint and GH joint act togheter in arm elevation according to scapularhumeral rhythm described in [99]. From a biomechanical point of view, shoulder complexity is justified by the high degree of coupling and coordination between shoulder joints (i.e., shoulder rhythm) and the action of more than one muscles over more than one joints in the execution of a movement. Data extraction of shoulder kinematics is frequently based on movements pattern in the sagittal, frontal and transversal planes, so monitoring of complex movements (e.g., daily activities) in multiple planes, performed through wearable sensors, requires a more stringent evaluation and accurate interpretation. As resulted in the review, the shoulder is generally approximated as a ball-and-socket joint [56]. This assumption provides an approximate representation of the whole shoulder girdle (e.g., it neglects the contribution of scapular movements). A standardized protocol has been proposed (i.e., The ISEO ${ }^{\circ}$, INAIL Shoulder and Elbow Outpatient protocol) to improve the performance of M-IMUs in the estimation of scapular kinematics, by locating inertial sensors on the back in correspondence of scapula [33, 35-38, 40, 65]. An adequate investigation of scapular motions may be beneficial to assess shoulder disorders [100].

For long-term monitoring of shoulder kinematics considering also scapular motions, the combination of MIMUs and smart-textile with embedded strain sensors is a perfect balancing of accuracy, flexibility and wearability (i.e., strain sensors positioned on the scapula could increase the portability and acceptance of the wearable system for long-term monitoring of ADLs) [86].

\section{Applicability in clinical setting and rehabilitation}

Alterations in the complex shoulder kinematics can derive by both neurological or musculoskeletal disorders and result in pain and limited movements [68]. Compensatory movements in patients with shoulder disorders are the most common consequential responses to pain or to difficulty in performing free-pain movements. In such situations, information retrieved by posture monitoring may be beneficial in clinical application and rehabilitation [26]. In the last years, the application of wearable devices for gathering motion data outside the laboratory settings is growing. Avoiding complex laboratory set-up, wearable systems employed to assess upper limb kinematics have proven to be a well-founded alternative to obtain quantitative motions parameters. Quantitative outcomes about shoulder motions recorded by wearable sensors are beneficial in clinical practice in terms of time-saving and they are becoming a promising alternative to improve assessment accuracy overcoming the subjectivity of clinical scales. The automatic assessment of motor abilities can also provide therapists a tangible and, therefore, measurable awareness of the effectiveness of the treatment and the recovery path chosen.

In clinical practice, the severity level of patients' condition with musculoskeletal disease is usually assessed through questionnaire-based scores [36, 42]. Algorithms for kinematic scores computing were developed to 


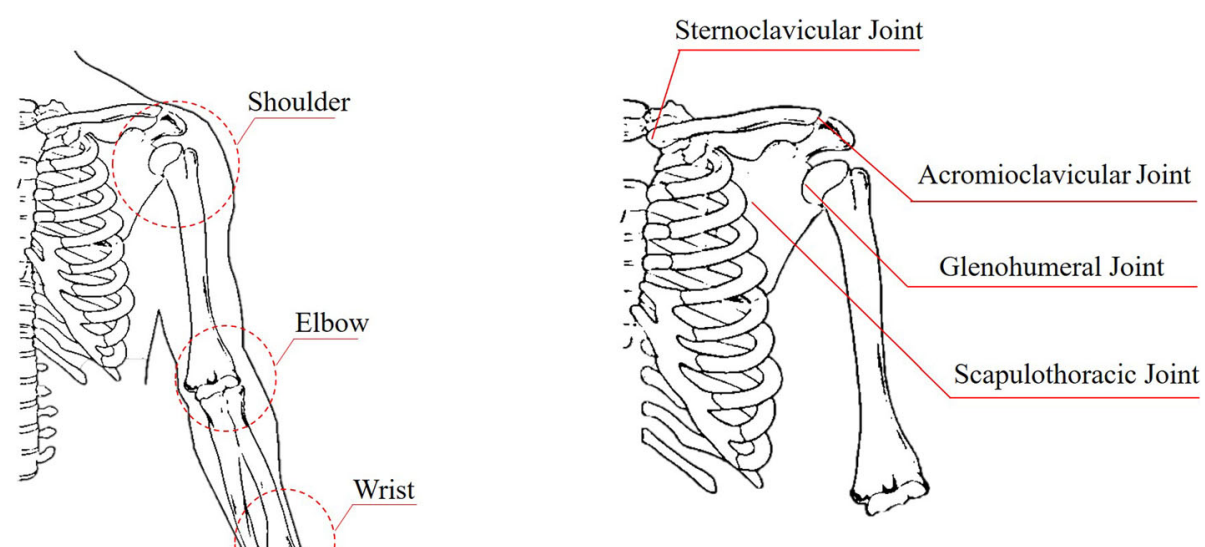

Fig. 3 a Anatomy of the Upper limb; b Anatomy of the Shoulder complex

evaluate shoulder functional performance after surgery in subjects with GH osteoarthritis and RC diseases, elaborating data obtained from IMU sensors [29, 31]. High correlation (0.61-0.8) between shoulder kinematic scores (i.e., power score, range of angular velocity score and moment score) and clinical scales (e.g., DASH, SST, VAS) was found [31]. Unlike clinical scores, kinematic scores showed greater sensitivity in detecting significant functional changes in shoulder activity at each postoperative follow-up with respect to the baseline status $[29,31]$. In a five-year follow-up study, asymmetry in shoulder movements was evaluated in patients with subacromial impingements syndrome. Asymmetry scores, derived from an IMU-based system, showed posttreatment improvements with greater sensitivity than clinical scores and only a weak correlation was found with DASH $(r=0.39)$ and SST $(r=0.32)$ [32]. Quantitative evaluation of arm usage and quality of movements in every kind of shoulder impairment contributes to outline a clinical picture about the functional recovery and the effectiveness of the treatment [30, 49]. Using the same number of IMU $(n=3)$ and the same placement on both humeri and sternum, the shoulder function was evaluated before and after treatment, in patients underwent surgery for RC tear [5, 34]. Results showed significative differences in movements frequency between patients and control group during activities of daily life [5], with limited use of arm at 3 months after surgery [34]. With a bilateral configuration based on 5 IMU, shoulder motion was assessed to extrapolate relevant clinical outcomes about Total Shoulder Arthroplasty (TSA) and Reverse Total Shoulder Arthroplasty (RTSA) [6]. Patients underwent either TSA or RTSA showed shoulder ROM below $80^{\circ}$ of elevation, indiscriminately; but, on average, patients treated with RTSA performed movements above $100^{\circ}$ less frequently [6]. Objective measurements (i.e., mean activity value and activity frequency) of limb function after RTSA did not show significant improvements 1 year after surgery, despite DASH scores and pain perception have improved compared to preoperative outcomes [41].

In patient with neurological impairments (e.g., stroke), assessments of motor abilities performed through wearable sensors showed a time saving compared to clinical scores (e.g., Fugl-Meyer Assessment Test) measured by the clinician [50, 53]. Data from accelerometers-based systems demonstrated accurate capability in the estimation of clinical scores for quality of movement (e.g., FAS score) and in prediction of shoulder features about shoulder portion of Fugl-Meyer scale with errors near 10\% [42, 49]. Generally, the main evaluated features comprise coordination, smoothness, presence of compensatory movements, speed, amplitude of ROM. Quantitative measurements, such as movement time and smoothness, showed a strong correlation with Action research arm test scores in patients after stroke [7]. Spatiotemporal parameters (e.g., ROM, movement time) extracted from inertial sensors' data provided an accurate evaluation of patients with multiple sclerosis and they distinguished affected and unaffected upper limbs in children with hemiparesis significantly [60, 62].

Digital simulations and virtual reality implementation in upper limb rehabilitation context aim to reproduce accurately limb movements processing data from wearable sensors and give a direct feedback about the adequacy or not of the executed movements [40]. The long-term monitoring, associated with suitable feedback strategy (e.g., visive, auditory, vibrational), can foster the correction of wrong postures [40, 52]. In addition, 
wearable systems allows a more supervised homerehabilitation giving substantial improvements to patient healing: total patient involvement in rehabilitation programs can advantage the motor learning process and, at the same time, providing a direct feedback (e.g., visual, auditory) about performance level can increase patient interest and motivation $[44,48]$. A new trend is the use of smartphone as monitoring systems or user-interface $[53,76,84]$. Implementation of suitable application (i.e., App) can provide a direct feedback to the patients and therapists about the progress in motor performance [26]. Gathered data could be remotely evaluated by the therapists [64]. Remote monitoring can provide useful information about patients' status at every stage of rehabilitation pathway and, at the same time, it implies a greater centralization of patients role in the management of their own health associated to a more direct clinician control [101]. A typical architecture of remote monitoring systems includes: $i$ ) wearable sensing unit to gather movements data; ii) data storage and management in cloud computing; iii) software to analyse data and extract relevant clinical parameters $[58,66]$. This approach implies collection of big amounts of data regarding personal information that requires ethical considerations and the definition of legal responsibility [102].

Most of the reviewed articles limited the application of wearable systems in short-time session for shoulder motion evaluation; only few studies performed longer monitoring periods of ADLs until 7 or 11 monitoring hours of 1 day $[5,6,34]$.

\section{Conclusion}

This review reveals that wearable systems are becoming an efficient and promising tool to evaluate shoulder health after neurological trauma or musculoskeletal injuries. Wearable systems have the potential to provide quantitative and meaningful clinical information about movement quality and progress in a rehabilitation pathway. The magneto-inertial measurements systems resulted the most used in clinical and research settings, followed by the growing application of smart-textiles for joint angles assessment. Despite of the accuracy of the current wearable systems in shoulder kinematics assessment, additional investigation needs to be executed to ensure long-term applicability in clinical settings and rehabilitation.

\section{Abbreviations}

AB-AD: abduction-adduction; AC: acromioclavicular; CMS: Constant-Murley score; DASH: Disability of the Arm, Shoulder, and Hand; DOFs: degrees of freedom; EMG: electromyography; ER: external rotation; FLX-EXT: flexionextension; GH: glenohumeral; HT: humerothoracic; IE: internal rotation; IER: internal-external rotation; IMU: inertial measurement unit; KF: Kalman filter; MEMS: micro-electro-mechanical systems; M-IMU: magnetic and inertial measurement unit; OLE: Optical Linear Encoder; PRISMA: Preferred Reporting Items for Systematic Reviews and Meta-Analyses; RC: rotator cuff; RMSE: root mean square error; ROM: range of motion; RTSA: Reverse Total Shoulder Arthroplasty; SC: sternoclavicular; SST: Simple Shoulder test:

ST: scapulothoracic; TSA: Total Shoulder Arthroplasty; VAS: Visual Analogue Scale

Acknowledgements

Not applicable.

\section{Authors' contributions}

UGL and ES conceived and supervised the study. AC carried out the search process and data collection, UGL, ES and VD assessed the quality of the study. AC and ES drafted the manuscript. All the authors have read and approved the final manuscript.

\section{Funding}

This work has been funded by the Italian Ministry of Health in the framework of RICERCA FINALIZZATA 2016 (PE-2016-02364894).

Availability of data and materials

The datasets used and/or analysed during the current study available from the corresponding author on reasonable request.

Ethics approval and consent to participate Not applicable.

Consent for publication

Not applicable.

\section{Competing interests}

UGL and $A B$ are members of the Editorial Board of BMC Musculoskeletal Disorders. The remaining authors declare that they have no conflict of interest.

\section{Author details}

${ }^{1}$ Department of Orthopaedic and Trauma Surgery, Campus Bio-Medico University, Via Álvaro del Portillo, 200, 00128 Rome, Italy. ${ }^{2}$ Unit of Measurements and Biomedical Instrumentation, Campus Bio-Medico University, Via Álvaro del Portillo, 21, 00128 Rome, Italy.

Received: 5 July 2019 Accepted: 31 October 2019

Published online: 15 November 2019

\section{References}

1. Cutti AG, Veeger HE. Shoulder biomechanics: today's consensus and tomorrow's perspectives. Med Biol Eng Comput. 2009;47(5):463-6.

2. Longo UG, Vasta S, Maffulli N, Denaro V. Scoring systems for the functional assessment of patients with rotator cuff pathology. Sports Med Arthrosc Rev. 2011:19(3):310-20

3. Longo UG, Berton A, Ahrens PM, Maffulli N, Denaro V. Clinical tests for the diagnosis of rotator cuff disease. Sports Med Arthrosc Rev. 2011;19(3):26678.

4. Longo UG, Saris D, Poolman RW, Berton A, Denaro V. Instruments to assess patients with rotator cuff pathology: a systematic review of measurement properties. Knee Surg Sports Traumatol Arthrosc. 2012;20(10):1961-70.

5. Duc C, Farron A, Pichonnaz C, Jolles BM, Bassin JP, Aminian K. Distribution of arm velocity and frequency of arm usage during daily activity: objective outcome evaluation after shoulder surgery. Gait Posture. 2013;38(2):247-52.

6. Langohr GDG, Haverstock JP, Johnson JA, Athwal GS. Comparing daily shoulder motion and frequency after anatomic and reverse shoulder arthroplasty. J Shoulder Elb Surg. 2018;27(2):325-32.

7. Repnik E, Puh U, Goljar N, Munih M, Mihelj M. Using Inertial Measurement Units and Electromyography to Quantify Movement during Action Research Arm Test Execution. Sensors (Basel). 2018;18:9

8. Bartalesi R, Lorussi F, Tesconi M, Tognetti A, Zupone G, Rossi DD: Wearable kinesthetic system for capturing and classifying upper limb gesture. In: First Joint Eurohaptics Conference and Symposium on Haptic Interfaces for Virtual Environment and Teleoperator Systems World Haptics Conference: 18-20 March 2005 2005; 2005: 535-536.

9. Massaroni C, Di Tocco J, Presti DL, Schena E, Bressi F, Bravi M, Miccinilli S, Sterzi S, Longo UG, Berton A: Influence of motion artifacts on a smart garment for monitoring respiratory rate. In: 2019 IEEE International 
Symposium on Medical Measurements and Applications (MeMeA): 2019: IEEE 2019: 1-6.

10. Presti DL, Massaroni C, Di Tocco J, Schena E, Formica D, Caponero MA, Longo UG, Carnevale A, D'Abbraccio J, Massari L: Cardiac monitoring with a smart textile based on polymer-encapsulated FBG: influence of sensor positioning. In: 2019 IEEE International Symposium on Medical Measurements and Applications (MeMeA): 2019: IEEE; 2019: 1-6.

11. Esfahani MIM, Nussbaum MA. A "smart" undershirt for tracking upper body motions: task classification and angle estimation. IEEE Sensors J. 2018;18(18): 7650-8.

12. Jordan K, Haywood KL, Dziedzic K, Garratt AM, Jones PW, Ong BN, Dawes PT. Assessment of the 3-dimensional Fastrak measurement system in measuring range of motion in ankylosing spondylitis. J Rheumatol. 2004; 31(11):2207-15.

13. Illyés A, Kiss RM. Method for determining the spatial position of the shoulder with ultrasound-based motion analyzer. J Electromyogr Kinesiol. 2006;16(1):79-88.

14. Kuster RP, Heinlein B, Bauer CM, Graf ES. Accuracy of KinectOne to quantify kinematics of the upper body. Gait Posture. 2016;47:80-5

15. Pérez R, Costa Ú, Torrent M, Solana J, Opisso E, Cáceres C, Tormos JM, Medina J, Gómez EJ. Upper limb portable motion analysis system based on inertial technology for neurorehabilitation purposes. Sensors (Basel). 2010; 10(12):10733-51.

16. Lambrecht JM, Kirsch RF. Miniature low-power inertial sensors: promising technology for implantable motion capture systems. IEEE Trans Neural Syst Rehabil Eng. 2014;22(6):1138-47.

17. Fantozzi S, Giovanardi A, Magalhães FA, Di Michele R, Cortesi M, Gatta G. Assessment of three-dimensional joint kinematics of the upper limb during simulated swimming using wearable inertial-magnetic measurement units. Sports Sci. 2016;34(11):1073-80.

18. Presti DL, Massaroni C, Formica D, Saccomandi P, Giurazza F, Caponero MA, Schena E. Smart textile based on 12 fiber Bragg gratings array for vital signs monitoring. IEEE Sensors J. 2017;17(18):6037-43.

19. Massaroni C, Carraro E, Vianello A, Miccinilli S, Morrone M, Levai IK, Schena E, Saccomandi P, Sterzi S, Dickinson JW, et al. Optoelectronic Plethysmography in clinical practice and research: a review. Respiration. 2017;93(5):339-54.

20. Massaroni C, Venanzi C, Silvatti AP, Lo Presti D, Saccomandi P, Formica D, Giurazza F, Caponero MA, Schena E. Smart textile for respiratory monitoring and thoraco-abdominal motion pattern evaluation. J Biophotonics. 2018; 11(5):e201700263.

21. de Lucena DS, Stoller O, Rowe JB, Chan V, Reinkensmeyer DJ. Wearable sensing for rehabilitation after stroke: bimanual jerk asymmetry encodes unique information about the variability of upper extremity recovery. IEEE Int Conf Rehabil Robot. 2017;2017:1603-8.

22. Patel $S$, Park $H$, Bonato $P$, Chan L, Rodgers M. A review of wearable sensors and systems with application in rehabilitation. Journal of neuroengineering and rehabilitation. 2012;9:21.

23. Muro-de-la-Herran A, Garcia-Zapirain B, Mendez-Zorrilla A. Gait analysis methods: an overview of wearable and non-wearable systems, highlighting clinical applications. Sensors (Basel). 2014;14(2):3362-94.

24. Bergmann JH, Chandaria V, McGregor A. Wearable and implantable sensors: the patient's perspective. Sensors (Basel). 2012;12(12):16695-709.

25. Caldani L, Pacelli M, Farina D, Paradiso R. E-textile platforms for rehabilitation. Conf Proc IEEE Eng Med Biol Soc. 2010;2010:5181-4.

26. Wang Q, De Baets L, Timmermans A, Chen W, Giacolini L, Matheve T, Markopoulos P. Motor Control Training for the Shoulder with Smart Garments. Sensors (Basel). 2017;17:7.

27. Burns DM, Leung N, Hardisty M, Whyne CM, Henry P, McLachlin S. Shoulder physiotherapy exercise recognition: machine learning the inertial signals from a smartwatch. Physiol Meas. 2018;39(7):075007.

28. Liberati A, Altman DG, Tetzlaff J, Mulrow C, Gøtzsche PC, loannidis JP, Clarke M, Devereaux PJ, Kleijnen J, Moher D. The PRISMA statement for reporting systematic reviews and meta-analyses of studies that evaluate health care interventions: explanation and elaboration. J Clin Epidemiol. 2009;62(10):e1-34.

29. Coley B, Jolles BM, Farron A, Bourgeois A, Nussbaumer F, Pichonnaz C, Aminian K. Outcome evaluation in shoulder surgery using 3D kinematics sensors. Gait Posture. 2007;25(4):523-32.

30. Coley B, Jolles BM, Farron A, Pichonnaz C, Bassin JP, Aminian K. Estimating dominant upper-limb segments during daily activity. Gait Posture. 2008; 27(3):368-75.
31. Jolles BM, Duc C, Coley B, Aminian K, Pichonnaz C, Bassin JP, Farron A. Objective evaluation of shoulder function using body-fixed sensors: a new way to detect early treatment failures? J Shoulder Elb Surg. 2011;20(7):1074-81.

32. Körver RJ, Senden R, Heyligers IC, Grimm B. Objective outcome evaluation using inertial sensors in subacromial impingement syndrome: a five-year follow-up study. Physiol Meas. 2014;35(4):677-86.

33. van den Noort JC, Wiertsema SH, Hekman KMC, Schönhuth CP, Dekker J, Harlaar J. Reliability and precision of 3D wireless measurement of scapular kinematics. Med Biol Eng Comput. 2014;52(11):921-31.

34. Pichonnaz C, Duc C, Jolles BM, Aminian K, Bassin JP, Farron A. Alteration and recovery of arm usage in daily activities after rotator cuff surgery. J Shoulder Elb Surg. 2015;24(9):1346-52.

35. Roldán-Jiménez C, Cuesta-Vargas Al. Studying upper-limb kinematics using inertial sensors: a cross-sectional study. BMC Res Notes. 2015;8:532.

36. van den Noort JC, Wiertsema SH, Hekman KM, Schönhuth CP, Dekker J, Harlaar J. Measurement of scapular dyskinesis using wireless inertial and magnetic sensors: importance of scapula calibration. J Biomech. 2015:48(12): 3460-8.

37. Cutti AG, Giovanardi A, Rocchi L, Davalli A, Sacchetti R. Ambulatory measurement of shoulder and elbow kinematics through inertial and magnetic sensors. Med Biol Eng Comput. 2008;46(2):169-78.

38. Roldán-Jiménez C, Cuesta-Vargas Al. Age-related changes analyzing shoulder kinematics by means of inertial sensors. Clin Biomech (Bristol, Avon). 2016;37:70-6

39. Aslani N, Noroozi S, Davenport P, Hartley R, Dupac M, Sewell P. Development of a 3D workspace shoulder assessment tool incorporating electromyography and an inertial measurement unit-a preliminary study. Med Biol Eng Comput. 2018;56(6):1003-11.

40. Carbonaro N, Lucchesi I, Lorusssi F, Tognetti A: Tele-monitoring and telerehabilitation of the shoulder muscular-skeletal diseases through wearable systems. In: 2018 40th Annual International Conference of the IEEE Engineering in Medicine and Biology Society (EMBC): 18-21 July 2018 2018; 2018: 44104413.

41. Hurd WJ, Morrow MM, Miller EJ, Adams RA, Sperling JW, Kaufman KR. Patient-reported and objectively measured function before and after reverse shoulder Arthroplasty. J Geriatr Phys Ther. 2018:41(3):126-33.

42. Hester T, Hughes R, Sherrill DM, Knorr B, Akay M, Stein J, Bonato P: Using wearable sensors to measure motor abilities following stroke. In: International Workshop on Wearable and Implantable Body Sensor Networks (BSN'06): 3-5 April 2006 2006; 2006: 4 pp.-8.

43. Zhou H, Hu H, Harris ND, Hammerton J. Applications of wearable inertial sensors in estimation of upper limb movements. Biomedical Signal Processing and Control. 2006;1(1):22-32

44. Willmann RD, Lanfermann G, Saini P, Timmermans A, te Vrugt J, Winter S. Home stroke rehabilitation for the upper limbs. Conf Proc IEEE Eng Med Biol Soc. 2007;2007:4015-8.

45. Zhou H, Stone T, Hu H, Harris N. Use of multiple wearable inertial sensors in upper limb motion tracking. Med Eng Phys. 2008;30(1):123-33.

46. Giorgino T, Tormene P, Lorussi F, Rossi DD, Quaglini S. Sensor evaluation for wearable strain gauges in neurological rehabilitation. IEEE Transactions on Neural Systems and Rehabilitation Engineering. 2009;17(4):409-15.

47. Lee GX, Low KS, Taher T. Unrestrained measurement of arm motion based on a wearable wireless sensor network. IEEE Trans Instrum Meas. 2010;59(5): 1309-17.

48. Chee Kian L, Chen I, Zhiqiang L, Yeo SH: A low cost wearable wireless sensing system for upper limb home rehabilitation. In: 2010 IEEE Conference on Robotics, Automation and Mechatronics: 28-30 June 2010 2010; 2010: 1-8.

49. Patel S, Hughes R, Hester T, Stein J, Akay M, Dy JG, Bonato P. A novel approach to monitor rehabilitation outcomes in stroke survivors using wearable technology. Proc IEEE. 2010;98(3):450-61.

50. Bento VF, Cruz VT, Ribeiro DD, Cunha JPS: Towards a movement quantification system capable of automatic evaluation of upper limb motor function after neurological injury. In: 2011 Annual International Conference of the IEEE Engineering in Medicine and Biology Society: 30 Aug.-3 Sept. 2011 2011; 2011: 5456-5460.

51. Nguyen KD, Chen I, Luo Z, Yeo SH, Duh HB. A wearable sensing system for tracking and monitoring of functional arm movement. IEEE/ASME Transactions on Mechatronics. 2011;16(2):213-20.

52. Ding ZQ, Luo ZQ, Causo A, Chen IM, Yue KX, Yeo SH, Ling KV. Inertia sensor-based guidance system for upperlimb posture correction. Med Eng Phys. 2013;35(2):269-76. 
53. Lee WW, Yen SC, Tay A, Zhao Z, Xu TM, Ling KK, Ng YS, Chew E, Cheong AL, Huat GK. A smartphone-centric system for the range of motion assessment in stroke patients. IEEE J Biomed Health Inform. 2014;18(6):183947.

54. Bai L, Pepper MG, Yan Y, Spurgeon SK, Sakel M, Phillips M. Quantitative assessment of upper limb motion in neurorehabilitation utilizing inertial sensors. IEEE Trans Neural Syst Rehabil Eng. 2015;23(2):232-43.

55. Ertzgaard P, Öhberg F, Gerdle B, Grip H. A new way of assessing arm function in activity using kinematic exposure variation analysis and portable inertial sensors--a validity study. Man Ther. 2016;21:241-9.

56. Lorussi F, Carbonaro N, De Rossi D, Tognetti A. A bi-articular model for scapular-humeral rhythm reconstruction through data from wearable sensors. J Neuroeng Rehabil. 2016;13:40.

57. Mazomenos EB, Biswas D, Cranny A, Rajan A, Maharatna K, Achner J, Klemke J, Jöbges M, Ortmann S, Langendörfer P. Detecting elementary arm movements by tracking upper limb joint angles with MARG sensors. IEEE Journal of Biomedical and Health Informatics. 2016;20(4):1088-99.

58. Jiang $Y$, Qin Y, Kim I, Wang Y: Towards an loT-based upper limb rehabilitation assessment system. In: 2017 39th Annual International Conference of the IEEE Engineering in Medicine and Biology Society (EMBC): 11-15 July 2017 2017; 2017: 2414-2417.

59. Li Y, Zhang X, Gong Y, Cheng Y, Gao X, Chen X. Motor Function Evaluation of Hemiplegic Upper-Extremities Using Data Fusion from Wearable Inertial and Surface EMG Sensors. Sensors (Basel). 2017;17:3.

60. Newman CJ, Bruchez R, Roches S, Jequier Gygax M, Duc C, Dadashi F, Massé F, Aminian K. Measuring upper limb function in children with hemiparesis with 3D inertial sensors. Childs Nerv Syst. 2017;33(12):2159-68.

61. Yang X, Tan J: Tracking of Human Joints Using Twist and Exponential Map. In: 2017 IEEE 7th Annual International Conference on CYBER Technology in Automation, Control, and Intelligent Systems (CYBER): 31 July-4 Aug. 2017 2017; 2017: 592-597.

62. Daunoraviciene K, Ziziene J, Griskevicius J, Pauk J, Ovcinikova A, Kizlaitiene R, Kaubrys G. Quantitative assessment of upper extremities motor function in multiple sclerosis. Technol Health Care. 2018;26(S2):647-53.

63. Jung H, Park J, Jeong J, Ryu T, Kim Y, Lee Sl: A wearable monitoring system for at-home stroke rehabilitation exercises: A preliminary study. In: 2018 IEEE EMBS International Conference on Biomedical \& Health Informatics (BHI): 4-7 March 2018 2018; 2018: 13-16.

64. Lin LF, Lin YJ, Lin ZH, Chuang LY, Hsu WC, Lin YH. Feasibility and efficacy of wearable devices for upper limb rehabilitation in patients with chronic stroke: a randomized controlled pilot study. Eur J Phys Rehabil Med. 2018; 54(3):388-96.

65. Parel I, Cutti AG, Fiumana G, Porcellini G, Verni G, Accardo AP. Ambulatory measurement of the scapulohumeral rhythm: intra- and inter-operator agreement of a protocol based on inertial and magnetic sensors. Gait Posture. 2012;35(4):636-40.

66. Daponte P, Vito LD, Sementa C: A wireless-based home rehabilitation system for monitoring 3D movements. In: 2013 IEEE International Symposium on Medical Measurements and Applications (MeMeA): 4-5 May 2013 2013; 2013: 282-287.

67. Daponte P, Vito LD, Sementa C: Validation of a home rehabilitation system for range of motion measurements of limb functions. In: 2013 IEEE International Symposium on Medical Measurements and Applications (MeMeA): 4-5 May 2013 2013; 2013: 288-293.

68. Pan J-I, Chung H-W, Huang J-J. Intelligent shoulder joint home-based selfrehabilitation monitoring system. Int J Smart Home. 2013;7(5):395-404.

69. Thiemjarus S, Marukatat S, Poomchoompol P. A method for shoulder rangeof-motion estimation using a single wireless sensor node. Conf Proc IEEE Eng Med Biol Soc. 2013;2013:5907-10.

70. Rawashdeh SA, Rafeldt DA, Uhl TL, Lumpp JE: Wearable motion capture unit for shoulder injury prevention. In: 2015 IEEE 12th International Conference on Wearable and Implantable Body Sensor Networks (BSN): 9-12 June 2015 2015; 2015: 1-6.

71. Álvarez D, Alvarez JC, González RC, López AM. Upper limb joint angle measurement in occupational health. Comput Methods Biomech Biomed Engin. 2016;19(2):159-70.

72. Lee $\mathrm{H}, \mathrm{Cho}$ J, Kim J: Printable skin adhesive stretch sensor for measuring multi-axis human joint angles. In: 2016 IEEE International Conference on Robotics and Automation (ICRA): 16-21 May 2016 2016; 2016: 4975-4980.

73. Tran TM, Vejarano G: Prediction of received signal strength from human joint angles in body area networks. In: 2016 International Conference on
Computing, Networking and Communications (ICNC): 15-18 Feb. 2016 2016; 2016: 1-6.

74. Rawashdeh SA, Rafeldt DA, Uhl TL. Wearable IMU for Shoulder Injury Prevention in Overhead Sports. Sensors (Basel). 2016;16:11.

75. Wu Y, Chen K, Fu C. Natural gesture modeling and recognition approach based on joint movements and arm orientations. IEEE Sensors J. 2016; 16(21):7753-61.

76. Ramkumar PN, Haeberle HS, Navarro SM, Sultan AA, Mont MA, Ricchetti ET, Schickendantz MS, lannotti JP. Mobile technology and telemedicine for shoulder range of motion: validation of a motion-based machine-learning software development kit. J Shoulder Elb Surg. 2018;27(7):1198-204.

77. Jung Y, Kang D, Kim J: Upper body motion tracking with inertial sensors. In: 2010 IEEE International Conference on Robotics and Biomimetics: 14-18 Dec. 2010 2010; 2010: 1746-1751.

78. El-Gohary M, Holmstrom L, Huisinga J, King E, McNames J, Horak F. Upper limb joint angle tracking with inertial sensors. Conf Proc IEEE Eng Med Biol Soc. 2011;2011:5629-32.

79. Zhang Z, Wong W, Wu J. Ubiquitous human upper-limb motion estimation using wearable sensors. IEEE Trans Inf Technol Biomed. 2011;15(4):513-21.

80. El-Gohary M, McNames J. Shoulder and elbow joint angle tracking with inertial sensors. IEEE Trans Biomed Eng. 2012;59(9):2635-41.

81. Lee GX, Low K. A factorized quaternion approach to determine the arm motions using Triaxial accelerometers with anatomical and sensor constraints. IEEE Trans Instrum Meas. 2012;61(6):1793-802.

82. Hsu Y, Wang J, Lin Y, Chen S, Tsai Y, Chu C, Chang C: A wearable inertialsensing-based body sensor network for shoulder range of motion assessment. In: 2013 1st International Conference on Orange Technologies (ICOT): 12-16 March 2013 2013; 2013: 328-331.

83. Ricci L, Formica D, Sparaci L, Lasorsa FR, Taffoni F, Tamilia E, Guglielmelli E. A new calibration methodology for thorax and upper limbs motion capture in children using magneto and inertial sensors. Sensors (Basel). 2014;14(1): 1057-72.

84. Roldan-Jimenez C, Cuesta-Vargas A, Bennett P. Studying upper-limb kinematics using inertial sensors embedded in Mobile phones. JMIR Rehabil Assist Technol. 2015;2(1):e4.

85. Meng D, Shoepe T, Vejarano G. Accuracy improvement on the measurement of human-joint angles. IEEE J Biomed Health Inform. 2016; 20(2):498-507.

86. Crabolu M, Pani D, Raffo L, Conti M, Crivelli P, Cereatti A. In vivo estimation of the shoulder joint center of rotation using magneto-inertial sensors: MRI-based accuracy and repeatability assessment. Biomed Eng Online. 2017;16(1):34.

87. Kim HJ, Lee YS, Kim D: Arm Motion Estimation Algorithm Using MYO Armband. In: 2017 First IEEE International Conference on Robotic Computing (IRC): 10-12 April 2017 2017; 2017: 376-381.

88. Morrow MMB, Lowndes B, Fortune E, Kaufman KR, Hallbeck MS. Validation of inertial measurement units for upper body kinematics. J Appl Biomech. 2017;33(3):227-32

89. Rose M, Curtze C, O'Sullivan J, El-Gohary M, Crawford D, Friess D, Brady JM. Wearable inertial sensors allow for quantitative assessment of shoulder and elbow kinematics in a cadaveric knee arthroscopy model. Arthroscopy. 2017:33(12):2110-6.

90. Tian Y, Li Y, Zhu L, Tan J: Inertial-based real-time human upper limb tracking using twists and exponential maps in free-living environments. In: 2017 2nd International Conference on Advanced Robotics and Mechatronics (ICARM): 27-31 Aug. 2017 2017; 2017: 552-557.

91. Pathirana PN, Karunarathne MS, Williams GL, Nam PT, Durrant-Whyte H. Robust and accurate capture of human joint pose using an inertial sensor. IEEE Journal of Translational Engineering in Health and Medicine. 2018;6:1-11.

92. Filippeschi A, Schmitz N, Miezal M, Bleser G, Ruffaldi E, Stricker D. Survey of Motion Tracking Methods Based on Inertial Sensors: A Focus on Upper Limb Human Motion. Sensors (Basel). 2017;17:6.

93. Madgwick SO, Harrison AJ, Vaidyanathan A. Estimation of IMU and MARG orientation using a gradient descent algorithm. IEEE Int Conf Rehabil Robot. 2011;2011:5975346.

94. Atalay O, Kennon W. Knitted strain sensors: impact of design parameters on sensing properties. Sensors. 2014;14(3):4712-30.

95. Holm R: Electric contacts: theory and application: Springer Science \& Business Media; 2013.

96. Yang J, Feng X, Kim JH, Rajulu S. Review of biomechanical models for human shoulder complex. International Journal of Human Factors Modelling and Simulation. 2010;1(3):271-93. 
97. Liu Y, Zhang Y, Zeng M: Joint parameter estimation using Magneto and Inertial measurement units. In: 2017 36th Chinese Control Conference (CCC): 26-28 July 2017 2017; 2017: 2225-2230.

98. Chen X, Zhang J, Hamel WR, Tan J: An inertial-based human motion tracking system with twists and exponential maps. In: 2014 IEEE International Conference on Robotics and Automation (ICRA): 31 May-7 June 2014 2014: 2014: 5665-5670.

99. Sugamoto K, Harada T, Machida A, Inui H, Miyamoto T, Takeuchi E, Yoshikawa H, Ochi T. Scapulohumeral rhythm: relationship between motion velocity and rhythm. Clin Orthop Relat Res. 2002;401:119-24.

100. Struyf F, Nijs J, Baeyens JP, Mottram S, Meeusen R. Scapular positioning and movement in unimpaired shoulders, shoulder impingement syndrome, and glenohumeral instability. Scand J Med Sci Sports. 2011;21(3):352-8.

101. Beckmann JS, Lew D. Reconciling evidence-based medicine and precision medicine in the era of big data: challenges and opportunities. Genome Med. 2016;8(1):134.

102. Sikka RS, Baer M, Raja A, Stuart M, Tompkins M. Analytics in sports medicine: implications and responsibilities that accompany the era of big data. J Bone Joint Surg Am. 2019;101(3):276-83.

\section{Publisher's Note}

Springer Nature remains neutral with regard to jurisdictional claims in published maps and institutional affiliations.

Ready to submit your research? Choose BMC and benefit from:

- fast, convenient online submission

- thorough peer review by experienced researchers in your field

- rapid publication on acceptance

- support for research data, including large and complex data types

- gold Open Access which fosters wider collaboration and increased citations

- maximum visibility for your research: over $100 \mathrm{M}$ website views per year

At $\mathrm{BMC}$, research is always in progress.

Learn more biomedcentral.com/submissions 